\title{
Infrequently expressed miRNAs influence survival after diagnosis with colorectal cancer
}

\section{Martha L. Slattery ${ }^{1}$, Andrew J. Pellatt ${ }^{2}$, Frances Y. Lee ${ }^{2}$, Jennifer S. Herrick ${ }^{3}$, Wade} S. Samowitz ${ }^{4}$, John R. Stevens ${ }^{5}$, Roger K. Wolff ${ }^{6}$ and Lila E. Mullany ${ }^{7}$

${ }^{1}$ Department of Medicine, University of Utah, Salt Lake City, Utah, USA

${ }^{2}$ Tulane Medical School, New Orleans, Louisiana, USA

${ }^{3}$ Department of Medicine, University of Utah, Salt Lake City, Utah, USA

${ }^{4}$ Department of Pathology, University of Utah, Salt Lake City, Utah, USA

${ }^{5}$ Department of Mathematics and Statistics, Utah State University, Logan, Utah, USA

${ }^{6}$ Department of Medicine, University of Utah, Salt Lake City, Utah, USA

${ }^{7}$ Department of Medicine, University of Utah, Salt Lake City, Utah, USA

Correspondence to: Martha L. Slattery, email: marty.slattery@hsc.utah.edu

Keywords: colorectal cancer, miRNAs, survival, stage, prognosis

Received: May 16, 2017 Accepted: July 25, $2017 \quad$ Published: August 03, 2017

Copyright: Slattery et al. This is an open-access article distributed under the terms of the Creative Commons Attribution License 3.0 (CC BY 3.0), which permits unrestricted use, distribution, and reproduction in any medium, provided the original author and source are credited.

\section{ABSTRACT}

Half of miRNAs expressed in colorectal tissue are expressed $<50 \%$ of the population. Many infrequently expressed miRNAs have low levels of expression. We hypothesize that less frequently expressed miRNAs, when expressed at higher levels, influence both disease stage and survival after diagnosis with colorectal cancer (CRC); low levels of expression may be background noise.

We examine 304 infrequently expressed miRNAs in 1893 population-based cases of CRC with paired carcinoma and normal mucosa miRNA profiles. We evaluate miRNAs with disease stage and survival after adjusting for age, study center, sex, MSI status, and AJCC stage. These miRNAs were further evaluated with RNA-Seq data to identify miRNA: :mRNA associations that may provide insight into the functionality of miRNAs.

Eleven miRNAs were associated with advanced disease stage among colon cancer patients $(Q$ value $=0.10)$. Eight infrequently expressed miRNAs influenced survival if highly expressed in overall CRC. Of these, five increased likelihood of dying if they were highly expressed, i.e. miR-124-3p, miR-143-5p, miR-145-3p, miR31-5p, and miR-99b-5p, while three were associated with better survival if highly expressed, i.e. miR-362-5p, miR-374a-5p, and miR-590-5p. Thirteen miRNAs infrequently expressed in colon-specific carcinoma tissue were associated with CRC survival if highly expressed. Evaluation of miRNAs::mRNA associations showed that mRNA expression influenced by infrequently expressed miRNA contributed to networks and pathways shown to influence disease progression and prognosis.

Our large study enabled us to examine the implications of infrequently expressed miRNAs after removal of background noise. These results require replication in other studies. Confirmation of our findings in other studies could lead to important markers for prognosis. 


\section{INTRODUCTION}

MiRNAs are small, non-protein-coding RNA molecules involved in the regulation of gene expression either by post-transcriptionally suppressing mRNA translation or by causing mRNA degradation [1-6]. Since associations between miRNA expression and colorectal cancer (CRC) were initially reported, $[7,8]$ over 2000 miRNAs have been identified and are available for study. Of these miRNAs, roughly 1300 are expressed in colorectal tissue. Most reported studies have small sample sizes and focus on miRNAs expressed commonly in the population. However, of those miRNAs expressed in CRC tissue, roughly $48 \%$ are expressed in less than half of the population [9]. These infrequently expressed miRNAs are most likely a mixture of those that are expressed at low levels, near the level of detection (background "noise"), and miRNAs that are potentially biologically relevant when expressed at higher levels although expressed less frequently in the population. Infrequently expressed miRNAs with higher levels of expression may be important for determining specific molecular pathways or to assist in identifying markers for disease progression and survival.

We have previously assessed miRNAs as they relate to CRC and survival $[10,11]$. We have shown that miRNAs appear to have a greater impact for survival after diagnosis with rectal cancer than with colon cancer [11]. Our main focus in previous analyses has been on commonly expressed miRNAs although we have examined the impact of "any" expression rather for miRNAs expressed less frequently, not taking into consideration potential background noise. While we detected some associations between survival and any expression of a few miRNAs, [11] it is possible that other infrequently expressed miRNAs are important only if expressed at higher levels. We have previously shown that infrequently expressed miRNAs are useful in distinguishing specific tumor molecular phenotypes [12].

In this study, we test the hypothesis that higher levels of expression of less frequently expressed miRNAs contribute to survival after a diagnosis of CRC. We also evaluate these infrequently expressed miRNAs with AJCC stages and site-specific CRC, i.e. colon or rectal cancer, associations. To gain insight into the functionality of these miRNAs, we utilize RNA-Seq data for mRNA expression to determine relationships between the miRNAs and their associated mRNAs. Our large sample size is essential to examine the effects of higher levels of expression of less frequently expressed miRNAs on disease progression and prognosis.

\section{RESULTS}

The population was predominately male with equal proportions of proximal and distal colon (Table 1). The majority of cases were diagnosed at AJCC stages 1 and 2. Out of a total of 2006 miRNAs assessed, 1278 or $63.7 \%$ were expressed in colorectal tumor tissue. Of those miRNAs expressed in CRC tissue, $47.8 \%$ were expressed in less than $50 \%$ of the study population with $72 \%$ being expressed in less than $10 \%$ of the study population.

Eleven infrequently expressed miRNAs were associated with stage when assessing colon cancer cases (Table 2). Of these 11 miRNAs, the up-regulation of 10 made a more advanced disease stage more likely than a lower disease stage. Up-regulation of one miRNA, miR-1244 was associated with a lower disease stage. The majority of these miRNAs were expressed at high levels in less than $20 \%$ of the population. For rectal cancer, there were nine miRNAs associated with disease stage prior to adjustment for multiple comparisons (Supplementary Table 2).

Assessment of infrequently expressed miRNAs in overall colorectal tumors with survival showed that eight miRNAs influenced survival when up-regulated in tumors (Table 3). Of these, five increased the likelihood of dying if they were up-regulated, i.e. miR-124-3p, miR-143-5p, miR-145-3p, miR31-5p, and miR-99b-5p, while three improved survival if up-regulated, i.e. miR-miR-362-5p, miR-374a-5p, and miR-590-5p. As with AJCC stage data, these miRNAs were infrequently highly expressed, except for miR-99b-3p, whose high differential expression was observed for $34 \%$ of the population. This high differential is from lack of expression in normal tissue, but higher levels of expression in carcinoma tissue.

Evaluation of site-specific associations with survival, showed that thirteen miRNAs infrequently expressed in colon cancer-specific tissue were associated with CRC survival if up-regulated (Table 4). Eleven of these miRNAs were associated with a greater likelihood of dying when up-regulated, while two miRNAs, miR632 and miR-362-5p, were associated with better survival if up-regulated. For rectal cancer, 26 miRNAs were associated with survival prior to adjustment for multiple comparisons (data not shown in table). Of these, 11 had a $Q$ value of 0.25 or less implying that potentially $25 \%$ of the findings were false positives. Of those miRNAs associated with site-specific survival prior to adjustment for multiple comparisons, only four miRNAs were associated with survival for both colon and rectal cancers, miR-124-3p, miR-2278, miR-4300, and miR-548aa. Of these, miR-548aa and miR-2278 were associated with increased likelihood of dying if upregulated in colon tissue while increasing the likelihood of survival if highly up-regulated in rectal tissue. After adjustment for multiple comparisons in rectal tumors, the $Q$ values were higher than 0.15 (Supplementary Table 3 shows results for rectal cancer). Results were similar with adjustment for MSI, although there was slightly less power (Supplementary Table 4 shows results adjusted for MSI). 
Table 1: Description of study population

\begin{tabular}{|c|c|c|c|c|c|c|c|}
\hline & & \multicolumn{2}{|c|}{ Overall } & \multicolumn{2}{|c|}{ Colon } & \multicolumn{2}{|c|}{ Rectal } \\
\hline & & Subject $N$ & $\%$ & Subject $N$ & $\%$ & Subject & $\%$ \\
\hline \multirow[t]{2}{*}{ Sex } & Male & 1027 & 54.3 & 607 & 52.8 & 420 & 56.5 \\
\hline & Female & 866 & 45.7 & 543 & 47.2 & 323 & 43.5 \\
\hline \multirow[t]{2}{*}{ Center } & Kaiser & 1144 & 60.4 & 740 & 64.3 & 404 & 54.4 \\
\hline & Utah & 749 & 39.6 & 410 & 35.7 & 339 & 45.6 \\
\hline \multirow[t]{2}{*}{ Site } & Proximal Colon & 581 & 50.5 & 581 & 50.5 & 0 & 0.0 \\
\hline & Distal Colon & 569 & 49.5 & 569 & 49.5 & 0 & 0.0 \\
\hline \multirow[t]{2}{*}{ Vital Status } & Dead & 914 & 48.3 & 562 & 49.0 & 352 & 47.4 \\
\hline & Alive & 977 & 51.7 & 586 & 51.0 & 391 & 52.6 \\
\hline \multirow[t]{5}{*}{ AJCC Stage } & Stage I & 559 & 30.0 & 259 & 22.7 & 300 & 41.5 \\
\hline & Stage II & 489 & 26.3 & 350 & 30.7 & 139 & 19.2 \\
\hline & Stage III & 548 & 29.4 & 340 & 29.9 & 208 & 28.8 \\
\hline & Stage IV & 266 & 14.3 & 190 & 16.7 & 76 & 10.5 \\
\hline & & $\operatorname{miRNA~N}^{1}$ & $\%$ & miRNA N & $\%$ & miRNA N & $\%$ \\
\hline \multirow[t]{9}{*}{$\begin{array}{l}\text { Percent Expressing in carcinoma } \\
\text { tissue: }\end{array}$} & No Expression & 728 & 36.3 & 759 & 37.8 & 882 & 44.0 \\
\hline & Any Expression & 1278 & 63.7 & 1247 & 62.2 & 1124 & 56.0 \\
\hline & $0>$ to 10 & 441 & 34.5 & 412 & 33.0 & 298 & 26.5 \\
\hline & $>10$ to 20 & 57 & 4.5 & 55 & 4.4 & 43 & 3.8 \\
\hline & $>20$ to 30 & 39 & 3.1 & 41 & 3.3 & 38 & 3.4 \\
\hline & $>30$ to 40 & 39 & 3.1 & 40 & 3.2 & 33 & 2.9 \\
\hline & $>40$ to 50 & 35 & 2.7 & 35 & 2.8 & 37 & 3.3 \\
\hline & $>50 \%$ & 667 & 52.2 & 664 & 53.2 & 675 & 60.1 \\
\hline & & Mean & STD & Mean & STD & Mean & STD \\
\hline Age & & 64.2 & 10.2 & 65.4 & 9.5 & 62.3 & 11.0 \\
\hline
\end{tabular}

${ }^{1}$ miRNA expression in carcinoma tissue.

Evaluation of the 24 miRNAs most strongly associated with either survival or disease stage (from Tables 2-4) also showed significantly (FDR $<0.05)$ altered differential gene mRNA expression for 14 of the miRNAs examined (Table 5). Five of these miRNAs, miR1, miR-133a, miR-145-3p, miR-3622b-3p, and miR-6722$5 p$, were associated with fewer than five genes that were differentially expressed in colorectal tissue. Four of the miRNAs, miR-31-5p, miR-362-5p, miR-934, and miR99b-5p, were associated with expression of over 100 genes in colorectal tissue. Over 100 of the genes associated with these miRNAs were associated with two to four miRNAs. This can be seen also in the overlap in functions of genes associated with these miRNAs.

When we assessed IPA networks derived from genes that were associated with all miRNAs that influenced survival, 17 networks had a score of 25 or greater Table 6). Figure 1 visually depicts the genes that are up- or down-regulated in the infrequently expressed miRNAs for the top four networks. Network 1
(Nervous system development and function, organ morphology, and organismal development) had a score of 35 with 33 focus molecules from our dataset in that network. Both Network 2 (Lipid metabolism, Molecular Transport, and Small Molecule Biochemistry) and Network 3 (Cellular development, Connective Tissue Development and Function, Skeletal and Muscular System Development and Function) had a score of 33 and 32 focus molecules of which several genes previously associated with colorectal cancer including $R U N X, B M P, I G F 1 R$, and $F A P$. The NFאB complex was central to the 4th Network (Developmental Disorder, Hereditary Disorder, and Immunological Disease).

\section{DISCUSSION}

Our data suggest that some miRNAs, although infrequently expressed, when expressed at higher levels in tumors may impact tumor aggressiveness and prognosis. While we tested the effects of both up-regulation and 
Table 2: Associations between infrequently expressed miRNAs and AJCC stage for colon cancer

Stage 1 and 2 Stage 3 and 4

$P$-value $Q$-values

\begin{tabular}{|c|c|c|c|c|c|c|c|c|c|c|}
\hline \multirow{2}{*}{$\frac{\text { miRNA }}{\text { hsa-miR-1244 }}$} & \multirow{2}{*}{$\begin{array}{l}\text { miRNA Expression }^{1} \\
\text { Down-regulated }\end{array}$} & \multirow{2}{*}{$\frac{N}{6}$} & \multirow{2}{*}{$\begin{array}{l}\mathbf{\%} \\
1.0\end{array}$} & \multirow{2}{*}{$\frac{N}{5}$} & \multirow{2}{*}{$\begin{array}{c}\% \\
0.9\end{array}$} & \multirow{2}{*}{$\frac{\mathbf{O R}^{2}}{0.84}$} & \multicolumn{2}{|c|}{$(95 \% \mathrm{CI})$} & \multirow[b]{2}{*}{0.7761} & \multirow[b]{2}{*}{0.78} \\
\hline & & & & & & & $(0.25$ & 2.79) & & \\
\hline & Referent & 448 & 73.6 & 431 & 81.3 & 1.00 & & & & \\
\hline & Up-regulated & 155 & 25.5 & 94 & 17.7 & 0.63 & $(0.47$ & $0.84)$ & 0.0017 & 0.10 \\
\hline \multirow[t]{3}{*}{ hsa-miR-133b } & Down-regulated & 231 & 37.9 & 210 & 39.6 & 1.12 & $(0.87$ & 1.46) & 0.3773 & 0.76 \\
\hline & Referent & 340 & 55.8 & 260 & 49.1 & 1.00 & & & & \\
\hline & Up-regulated & 38 & 6.2 & 60 & 11.3 & 2.00 & $(1.29$ & $3.11)$ & 0.0021 & 0.10 \\
\hline \multirow[t]{3}{*}{ hsa-miR-143-5p } & Down-regulated & 33 & 5.4 & 35 & 6.6 & 1.24 & $(0.75$ & $2.05)$ & 0.3954 & 0.76 \\
\hline & Referent & 559 & 91.8 & 457 & 86.2 & 1.00 & & & & \\
\hline & Up-regulated & 17 & 2.8 & 38 & 7.2 & 2.64 & $(1.46$ & $4.75)$ & 0.0013 & 0.10 \\
\hline \multirow[t]{3}{*}{ hsa-miR-31-5p } & Down-regulated & 14 & 2.3 & 9 & 1.7 & 0.81 & $(0.35$ & 1.90) & 0.6262 & 0.78 \\
\hline & Referent & 511 & 83.9 & 415 & 78.3 & 1.00 & & & & \\
\hline & Up-regulated & 84 & 13.8 & 106 & 20.0 & 1.56 & $(1.14$ & 2.15) & 0.0056 & 0.10 \\
\hline \multirow[t]{3}{*}{ hsa-miR-4290 } & Down-regulated & 33 & 5.4 & 28 & 5.3 & 1.02 & $(0.60$ & 1.72) & 0.9474 & 0.95 \\
\hline & Referent & 530 & 87.0 & 437 & 82.5 & 1.00 & & & & \\
\hline & Up-regulated & 46 & 7.6 & 65 & 12.3 & 1.73 & $(1.16$ & 2.58) & 0.0076 & 0.10 \\
\hline \multirow[t]{3}{*}{ hsa-miR-4324 } & Down-regulated & 72 & 11.8 & 42 & 7.9 & 0.65 & $(0.43$ & $0.97)$ & 0.0374 & 0.62 \\
\hline & Referent & 470 & 77.2 & 394 & 74.3 & 1.00 & & & & \\
\hline & Up-regulated & 67 & 11.0 & 94 & 17.7 & 1.60 & $(1.13$ & $2.25)$ & 0.0078 & 0.10 \\
\hline \multirow[t]{3}{*}{ hsa-miR-466 } & Down-regulated & 16 & 2.6 & 21 & 4.0 & 1.64 & $(0.84$ & 3.19) & 0.1476 & 0.69 \\
\hline & Referent & 562 & 92.3 & 459 & 86.6 & 1.00 & & & & \\
\hline & Up-regulated & 31 & 5.1 & 50 & 9.4 & 2.01 & $(1.26$ & $3.20)$ & 0.0035 & 0.10 \\
\hline \multirow[t]{3}{*}{ hsa-miR-502-3p } & Down-regulated & 12 & 2.0 & 6 & 1.1 & 0.59 & $(0.22$ & 1.61) & 0.3046 & 0.73 \\
\hline & Referent & 569 & 93.4 & 477 & 90.0 & 1.00 & & & & \\
\hline & Up-regulated & 28 & 4.6 & 47 & 8.9 & 2.00 & $(1.23$ & $3.26)$ & 0.0051 & 0.10 \\
\hline \multirow[t]{3}{*}{ hsa-miR-645 } & Down-regulated & 6 & 1.0 & 5 & 0.9 & 1.04 & $(0.31$ & $3.46)$ & 0.9505 & 0.95 \\
\hline & Referent & 449 & 73.7 & 344 & 64.9 & 1.00 & & & & \\
\hline & Up-regulated & 154 & 25.3 & 181 & 34.2 & 1.51 & $(1.17$ & 1.96) & 0.0017 & 0.10 \\
\hline \multirow[t]{3}{*}{ hsa-miR-6722-5p } & Down-regulated & 10 & 1.6 & 11 & 2.1 & 1.32 & $(0.56$ & $3.15)$ & 0.5276 & 0.78 \\
\hline & Referent & 592 & 97.2 & 498 & 94.0 & 1.00 & & & & \\
\hline & Up-regulated & 7 & 1.1 & 21 & 4.0 & 3.61 & $(1.52$ & $8.58)$ & 0.0037 & 0.10 \\
\hline \multirow[t]{3}{*}{ hsa-miR-934 } & Down-regulated & 22 & 3.6 & 13 & 2.5 & 0.91 & $(0.45$ & 1.87) & 0.8038 & 0.80 \\
\hline & Referent & 282 & 46.3 & 183 & 34.5 & 1.00 & & & & \\
\hline & Up-regulated & 305 & 50.1 & 334 & 63.0 & 1.70 & $(1.33$ & 2.16) & $<.0001$ & 0.10 \\
\hline
\end{tabular}

${ }^{1}$ Down-regulated was defined as <-1.77 Agilent Relative Florescent Units (ARFU); the referent group was -1.77-2.08 ARFU; upregulated was miRNA expression above 2.08 ARFU.

${ }^{2}$ Adjusted for age, study center, and sex.

down-regulation, the only significant results in Tables 2-4 were for up-regulated miRNAs such that there were higher expression levels in tumors. We observed that these infrequently expressed miRNAs were associated with CRC survival overall as well as with CRC sitespecific survival. Several infrequently expressed miRNAs were more likely to have different levels of expression dependent on stage at diagnosis.

When interpreting these results, several aspects of the study need to be considered. One of the premises of the study is that low levels of expression in miRNAs infrequently expressed in the population is the result of 
Table 3: Associations between infrequently expressed miRNAs and overall survival after diagnosis with colorectal cancer

\begin{tabular}{|c|c|c|c|c|c|c|c|c|c|c|}
\hline \multirow{3}{*}{$\frac{\text { miRNA }}{\text { hsa-miR-124-3p }}$} & \multirow{3}{*}{$\frac{\text { miRNA Expression }^{1}}{\text { Down-regulated }}$} & \multirow{3}{*}{$\begin{array}{c}\text { Censored } \\
\boldsymbol{N} \\
70\end{array}$} & \multicolumn{4}{|c|}{ Died of CRC } & \multicolumn{4}{|c|}{$P$-value } \\
\hline & & & $\%$ & $N$ & $\%$ & $\mathbf{H R}^{2}$ & \multicolumn{2}{|c|}{$(95 \% \mathrm{CI})$} & \multirow{2}{*}{$\frac{\text { Raw }}{0.127}$} & \multirow{2}{*}{$\frac{Q}{0.58}$} \\
\hline & & & 5.4 & 54 & 9.5 & 1.25 & $(0.94$ & 1.66) & & \\
\hline & Referent & 1184 & 92 & 488 & 85.9 & 1.00 & & & & \\
\hline & Up-regulated & 33 & 2.6 & 26 & 4.6 & 1.93 & $(1.30$ & 2.86) & 0.001 & 0.15 \\
\hline \multirow[t]{3}{*}{ hsa-miR-143-5p } & Down-regulated & 73 & 5.7 & 31 & 5.5 & 0.78 & $(0.53$ & $1.12)$ & 0.179 & 0.58 \\
\hline & Referent & 1177 & 91.5 & 489 & 86.1 & 1.00 & & & & \\
\hline & Up-regulated & 37 & 2.9 & 48 & 8.5 & 1.70 & $(1.26$ & 2.29) & 0.001 & 0.15 \\
\hline \multirow[t]{3}{*}{ hsa-miR-145-3p } & Down-regulated & 29 & 2.3 & 18 & 3.2 & 1.22 & $(0.76$ & $1.97)$ & 0.408 & 0.61 \\
\hline & Referent & 1252 & 97.3 & 535 & 94.2 & 1.00 & & & & \\
\hline & Up-regulated & 6 & 0.5 & 15 & 2.6 & 3.44 & $(2.04$ & $5.78)$ & $<.0001$ & 0.15 \\
\hline \multirow[t]{3}{*}{ hsa-miR-31-5p } & Down-regulated & 19 & 1.5 & 7 & 1.2 & 0.64 & $(0.30$ & $1.36)$ & 0.245 & 0.59 \\
\hline & Referent & 1114 & 86.6 & 455 & 80.1 & 1.00 & & & & \\
\hline & Up-regulated & 154 & 12 & 106 & 18.7 & 1.38 & $(1.11$ & $1.70)$ & 0.003 & 0.15 \\
\hline \multirow[t]{3}{*}{ hsa-miR-362-5p } & Down-regulated & 27 & 2.1 & 11 & 1.9 & 0.64 & $(0.35$ & $1.17)$ & 0.148 & 0.58 \\
\hline & Referent & 912 & 70.9 & 439 & 77.3 & 1.00 & & & & \\
\hline & Up-regulated & 348 & 27 & 118 & 20.8 & 0.70 & $(0.57$ & $0.86)$ & 0.001 & 0.15 \\
\hline \multirow[t]{3}{*}{ hsa-miR-374a-5p } & Down-regulated & 16 & 1.2 & 7 & 1.2 & 0.72 & $(0.34$ & $1.54)$ & 0.4 & 0.61 \\
\hline & Referent & 996 & 77.4 & 478 & 84.2 & 1.00 & & & & \\
\hline & Up-regulated & 275 & 21.4 & 83 & 14.6 & 0.63 & $(0.50$ & $0.80)$ & $<0.0001$ & 0.15 \\
\hline \multirow[t]{3}{*}{ hsa-miR-590-5p } & Down-regulated & 171 & 13.3 & 79 & 13.9 & 0.93 & $(0.74$ & 1.19) & 0.578 & 0.62 \\
\hline & Referent & 963 & 74.8 & 448 & 78.9 & 1.00 & & & & \\
\hline & Up-regulated & 153 & 11.9 & 41 & 7.2 & 0.62 & $(0.45$ & $0.86)$ & 0.004 & 0.15 \\
\hline \multirow[t]{3}{*}{ hsa-miR-99b-5p } & Down-regulated & 289 & 22.5 & 121 & 21.3 & 1.01 & $(0.81$ & $1.27)$ & 0.935 & 0.94 \\
\hline & Referent & 634 & 49.3 & 254 & 44.7 & 1.00 & & & & \\
\hline & Up-regulated & 364 & 28.3 & 193 & 34 & 1.33 & $(1.10$ & $1.61)$ & 0.004 & 0.15 \\
\hline
\end{tabular}

${ }^{1}$ Down-regulated was defined as $<-1.77$ Agilent Relative Florescent Units (ARFU); the referent group was -1.77-2.08 ARFU; upregulated was miRNA expression above 2.08 ARFU.

${ }^{2}$ Adjusted for age, study center, sex, and AJCC Stage.

noise in the data. This could represent miRNAs that were near the level of detection. Thus, this study specifically looks at higher levels of expression for infrequently expressed miRNAs to determine their impact on disease stage and survival. However, the determination of higher level of expression was somewhat arbitrary. We utilized the distribution of differential expression in the study population to help set these cutpoints. The upper and lower cutpoints were set at the 25 th percentile and the 75th percentile of paired tumor-normal differential expression, beyond which they were considered downregulated in carcinoma relative to normal or up-regulated in carcinoma tissue relative to normal mucosa. We further limited the analysis to only those miRNAs that had at least 30 individuals showing expression in tumors in order to have sufficient power to examine survival. Setting lower cutpoints could have increased our power, although specificity in associations could have been lost.
We previously have shown that five miRNAs were associated with survival after a diagnosis with colon cancer when any expression was evaluated. Three of these miRNAs, miR-1, miR-145-3p and miR-31-5p decreased survival when analyzed for any expression as well as for higher levels of expression in tumors in this study. HRs were similar, although slightly stronger when evaluating any expression. Analysis looking at any expression had more power than we have here looking at only higher levels of expression in tumors. However, our analyses suggest that higher levels of expression in tumors may be needed to influence prognosis for most miRNAs. This analysis is more robust when assessing infrequently expressed miRNAs because it eliminates the background noise that is present when assessing any expression. We believe that the survival associations with these miRNAs are independent of other miRNAs associated with colorectal cancer survival. Our previous analysis 
Table 4: Infrequently expressed miRNAs associated with survival after diagnosis with colon cancer

\begin{tabular}{|c|c|c|c|c|c|c|c|c|c|c|}
\hline \multirow{3}{*}{$\frac{\text { miRNA }}{\text { hsa-miR-1 }}$} & \multirow{3}{*}{$\begin{array}{l}\text { miRNA Expression }^{1} \\
\text { Down-regulated }\end{array}$} & \multirow{3}{*}{$\begin{array}{c}\text { Censored } \\
N \\
33\end{array}$} & \multirow{3}{*}{$\frac{\%}{4.2}$} & \multicolumn{2}{|c|}{ Died of CRC } & \multirow{3}{*}{$\frac{\mathbf{H R}^{2}}{0.78}$} & \multicolumn{4}{|c|}{$P$-value } \\
\hline & & & & $N$ & $\%$ & & \multicolumn{2}{|c|}{$(95 \% \mathrm{CI})$} & \multirow{2}{*}{$\frac{\text { Raw }}{0.325}$} & \multirow{2}{*}{$\frac{\boldsymbol{Q}}{0.96}$} \\
\hline & & & & 18 & 5.2 & & $(0.48$ & $1.28)$ & & \\
\hline & Referent & 748 & 94.7 & 310 & 90.1 & 1.00 & & & & \\
\hline & Up-regulated & 9 & 1.1 & 16 & 4.7 & 1.97 & $(1.18$ & $3.28)$ & 0.009 & 0.15 \\
\hline \multirow[t]{3}{*}{ hsa-miR-124-3p } & Down-regulated & 55 & 7 & 39 & 11.3 & 1.15 & $(0.82$ & 1.61) & 0.427 & 1.00 \\
\hline & Referent & 713 & 90.3 & 287 & 83.4 & 1.00 & & & & \\
\hline & Up-regulated & 22 & 2.8 & 18 & 5.2 & 2.19 & $(1.35$ & $3.55)$ & 0.001 & 0.15 \\
\hline \multirow[t]{3}{*}{ hsa-miR-133a } & Down-regulated & 23 & 2.9 & 15 & 4.4 & 0.90 & $(0.53$ & $1.53)$ & 0.688 & 1.00 \\
\hline & Referent & 760 & 96.2 & 315 & 91.6 & 1.00 & & & & \\
\hline & Up-regulated & 7 & 0.9 & 14 & 4.1 & 1.98 & $(1.16$ & $3.40)$ & 0.013 & 0.15 \\
\hline \multirow[t]{3}{*}{ hsa-miR-143-5p } & Down-regulated & 48 & 6.1 & 20 & 5.8 & 0.69 & $(0.43$ & 1.10) & 0.119 & 0.91 \\
\hline & Referent & 720 & 91.1 & 291 & 84.6 & 1.00 & & & & \\
\hline & Up-regulated & 22 & 2.8 & 33 & 9.6 & 1.75 & $(1.22$ & 2.52) & 0.003 & 0.15 \\
\hline \multirow[t]{3}{*}{ hsa-miR-145-3p } & Down-regulated & 18 & 2.3 & 12 & 3.5 & 1.12 & $(0.62$ & 2.03) & 0.704 & 1.00 \\
\hline & Referent & 767 & 97.1 & 319 & 92.7 & 1.00 & & & & \\
\hline & Up-regulated & 5 & 0.6 & 13 & 3.8 & 3.21 & $(1.82$ & $5.64)$ & $<.0001$ & 0.15 \\
\hline \multirow[t]{3}{*}{ hsa-miR-31-5p } & Down-regulated & 18 & 2.3 & 5 & 1.5 & 0.56 & $(0.23$ & 1.37) & 0.203 & 0.91 \\
\hline & Referent & 659 & 83.4 & 264 & 76.7 & 1.00 & & & & \\
\hline & Up-regulated & 113 & 14.3 & 75 & 21.8 & 1.43 & $(1.10$ & 1.85) & 0.007 & 0.15 \\
\hline \multirow[t]{3}{*}{ hsa-miR-3622b-3p } & Down-regulated & 23 & 2.9 & 9 & 2.6 & 1.05 & $(0.54$ & $2.05)$ & 0.879 & 1.00 \\
\hline & Referent & 590 & 74.7 & 240 & 69.8 & 1.00 & & & & \\
\hline & Up-regulated & 177 & 22.4 & 95 & 27.6 & 1.41 & $(1.11$ & 1.79) & 0.005 & 0.15 \\
\hline \multirow[t]{3}{*}{ hsa-miR-362-5p } & Down-regulated & 16 & 2 & 7 & 2 & 0.59 & $(0.28$ & 1.27) & 0.176 & 0.91 \\
\hline & Referent & 585 & 74.1 & 275 & 79.9 & 1.00 & & & & \\
\hline & Up-regulated & 189 & 23.9 & 62 & 18 & 0.70 & $(0.53$ & $0.92)$ & 0.012 & 0.15 \\
\hline \multirow[t]{3}{*}{ hsa-miR-378g } & Down-regulated & 156 & 19.7 & 77 & 22.4 & 1.15 & $(0.89$ & 1.49) & 0.292 & 0.96 \\
\hline & Referent & 586 & 74.2 & 238 & 69.2 & 1.00 & & & & \\
\hline & Up-regulated & 48 & 6.1 & 29 & 8.4 & 1.86 & $(1.26$ & 2.74) & 0.002 & 0.15 \\
\hline \multirow[t]{3}{*}{ hsa-miR-548aw } & Down-regulated & 21 & 2.7 & 11 & 3.2 & 1.01 & $(0.55$ & 1.84) & 0.977 & 1.00 \\
\hline & Referent & 763 & 96.6 & 326 & 94.8 & 1.00 & & & & \\
\hline & Up-regulated & 6 & 0.8 & 7 & 2 & 2.97 & $(1.40$ & $6.34)$ & 0.005 & 0.15 \\
\hline \multirow[t]{3}{*}{ hsa-miR-632 } & Down-regulated & 142 & 18 & 52 & 15.1 & 0.74 & $(0.55$ & $0.99)$ & 0.045 & 0.91 \\
\hline & Referent & 528 & 66.8 & 248 & 72.1 & 1.00 & & & & \\
\hline & Up-regulated & 120 & 15.2 & 44 & 12.8 & 0.66 & $(0.48$ & $0.91)$ & 0.012 & 0.15 \\
\hline \multirow[t]{3}{*}{ hsa-miR-645 } & Down-regulated & 5 & 0.6 & 6 & 1.7 & 3.14 & $(1.37$ & 7.16) & 0.007 & 0.59 \\
\hline & Referent & 576 & 72.9 & 213 & 61.9 & 1.00 & & & & \\
\hline & Up-regulated & 209 & 26.5 & 125 & 36.3 & 1.32 & $(1.06$ & $1.65)$ & 0.014 & 0.15 \\
\hline \multirow[t]{3}{*}{ hsa-miR-671-3p } & Down-regulated & 42 & 5.3 & 22 & 6.4 & 1.27 & $(0.82$ & $1.96)$ & 0.277 & 0.96 \\
\hline & Referent & 727 & 92 & 310 & 90.1 & 1.00 & & & & \\
\hline & Up-regulated & 21 & 2.7 & 12 & 3.5 & 2.32 & $(1.30$ & $4.16)$ & 0.005 & 0.15 \\
\hline
\end{tabular}

${ }^{1}$ Down-regulated was defined as <-1.77 Agilent Relative Florescent Units (ARFU); the referent group was -1.77-2.08 ARFU; upregulated was miRNA expression above 2.08 ARFU.

${ }^{2}$ Adjusted for age, study center, sex, and AJCC Stage.

did not identify any miRNAs associated with colon cancer after adjustment for multiple comparisons [11], thus confounding is not likely an issue. For rectal cancer we identified several commonly expressed miRNAs that were associated with survival, while in this study we did not identify significant associations after adjustment for multiple comparisons. It is possible that lack of power is in part responsible for these lack of findings in this 


\section{Table 5: miRNAs associated with survival or disease stage $(Q$ value $<0.15)$, their targeted genes and associated pathways}

\begin{tabular}{|c|c|c|}
\hline miRNA & Genes associated with miRNAs in colorectal tissue using RNAseq mRNA expression data ${ }^{1}$ & IPA Top Diseases and Functions ${ }^{2}$ \\
\hline hsa-miR-1 & HANDI & \\
\hline hsa-miR-133a & CHRDL2, GRIK5 & \\
\hline hsa-miR-133b & $\begin{array}{l}\text { HSPB6, PER3, MYLK, PDZD4, LIMS2, SPEG, TACR2, TNS1, PGR, SORBS1, GRIK5, HAND1, LDB3, NECAB1, } \\
\text { CNN1, AOC3, MYH11, MEIS2, FXYD6, MYOCD, BOC, TAGLN, JPH2, GPM6A, SPARCL1, CACNA2D1, } \\
\text { KCNMA1, ADAMTSL3, ACTG2, LMOD1, LONRF2, SYNPO2, HSPB7, DES, CHRM2, MAB2IL2, SYNM, DACT3, } \\
\text { DMD, RPI1-728F11.6 }\end{array}$ & $\begin{array}{l}\text { Cancer, Organismal Injury and Abnormalities, Reproductive } \\
\text { System Disease }\end{array}$ \\
\hline hsa-miR-143-5p & $\begin{array}{l}\text { HSPB6, MYLK, TNS1, SORBS1, GRIK5, HAND1, NECAB1, CNN1, MYHI1, SPARCL1, KCNMA1, ACTG2, } \\
\text { LMOD1, SYNPO2, DES, CHRM2, SYNM, DACT3, PLN }\end{array}$ & $\begin{array}{l}\text { Cardiovascular System Development and } \text { Function, } \\
\text { Developmental Disorder, Embryonic Development }\end{array}$ \\
\hline hsa-miR-145-3p & HAND1, CHRM2 & \\
\hline hsa-miR-31-5p & 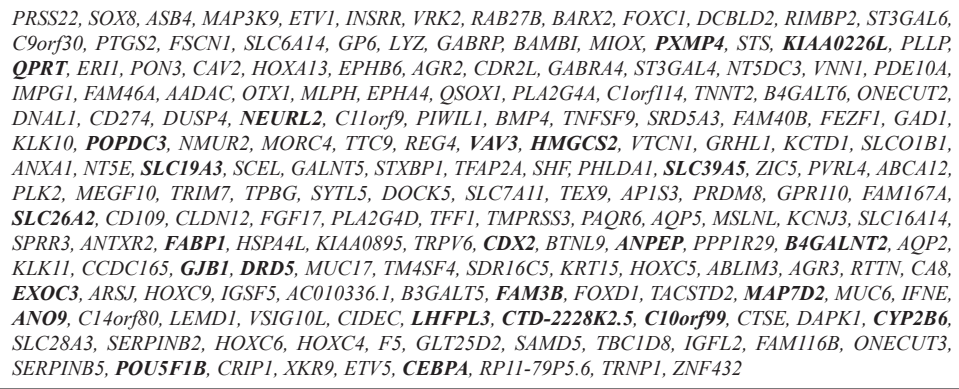 & $\begin{array}{l}\text { Cancer, Gastrointestinal Disease, Organismal injury and } \\
\text { Abnormalities; Cancer, Organismal injury and Abnormalities, } \\
\text { Immunological Diseases; Connective tissue Development and } \\
\text { Function, Connective Tissue Disorders, Organ Morphology; } \\
\text { Humoral Immune Response }\end{array}$ \\
\hline hsa-miR-3622b-3p & LHFPL4 & \\
\hline hsa-miR-362-5p & 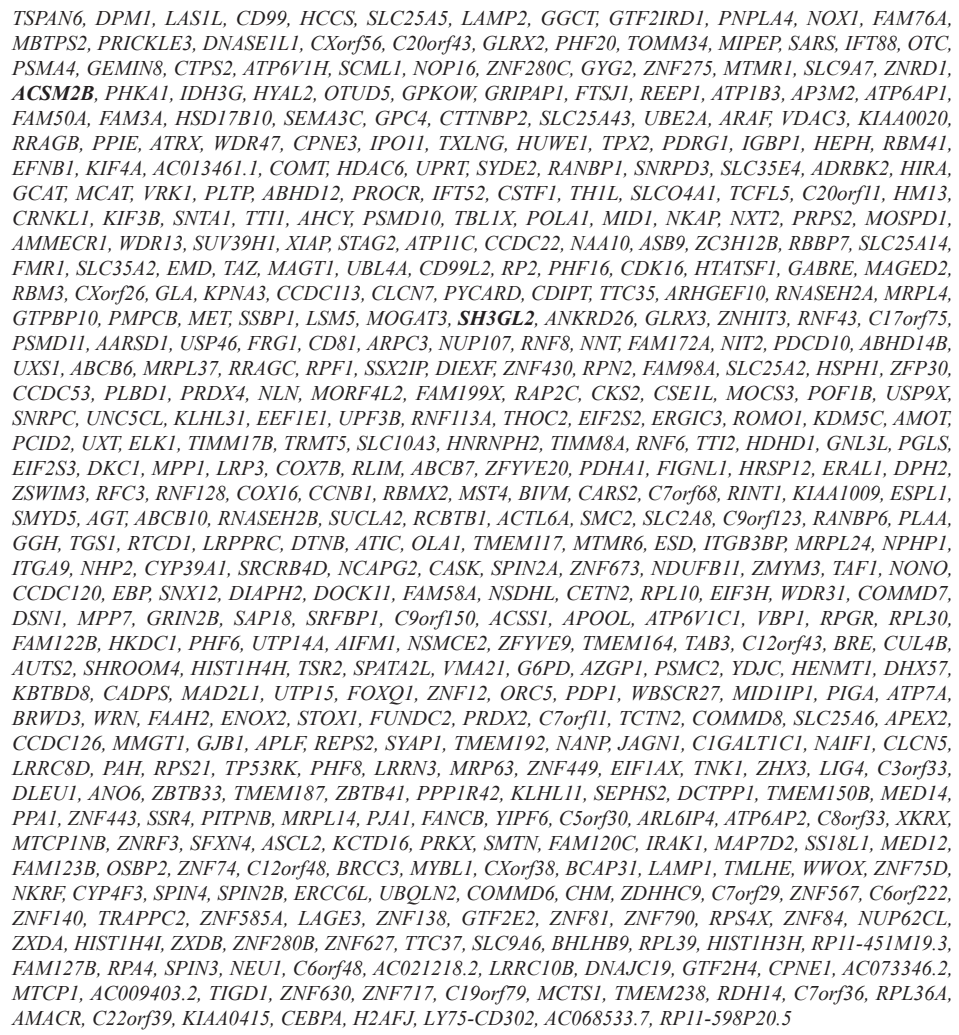 & $\begin{array}{l}\text { Cell Cycle, Cell Morphology, Cellular Assembly and } \\
\text { Organization; Developmental disorder, Hereditary Disorder, } \\
\text { Neurological Disease; Hereditary Disorder, Organismal } \\
\text { Injury and Abnormalities, Neurological Disease; Amino Acid } \\
\text { Metabolism, Cell Signaling, Cellular Function and Maintenance }\end{array}$ \\
\hline hsa-miR-374a-5p & $\begin{array}{l}\text { DPM1, LASIL, PNPLA4, ZFP64, C20orf43, GYG2, CDON, REEP1, CTTNBP2, BCORL1, PDRG1, PES1, } \\
\text { ABHD12, R3HDML, NDRG3, PABPCIL, PSMA7, KIF3B, MAPILC3A, MOSPD1, ZC3H12B, FMR1, PHF16, } \\
\text { GABRE, KRT23, PTK7, TGIF2, LYPLA1, PARD6B, MOCS3, EREG, ZC4H2, TTI2, ZNF337, DKC1, LRP3, } \\
\text { GGT7, RAI2, HRSP12, CTNNBL1, ZSWIM3, BEX2, APCDD1, WIF1, PHF6, SHROOM4, KALRN, BBS5, FOXQ1, } \\
\text { LARP6, GNG4, NANP, SCAND1, TP53RK, C2orf54, LRRN3, NCKAP5, LDLRAD3, YIPF6, FAM123B, ZNF74, } \\
\text { TMPRSS6, C11orf95, CHM, ZDHHC9, GSPT2, PCMTD2, CPNE1, C22orf29, EIF6 }\end{array}$ & $\begin{array}{l}\text { Cell Cycle, Cancer, Cell Death and Survival; Organismal } \\
\text { Survival, Cancer, Organismal Injury and Abnormalities }\end{array}$ \\
\hline hsa-miR-4324 & ANLN, CYBRD1, LIMS2, CHRDL1, MGP, CASQ2, FXYD6, BOC, CMYA5, RNF150, PODN, C7orf10, ZIM2 & \\
\hline hsa-miR-645 & $\begin{array}{l}\text { HECW1, MRC2, SLC11A1, TIMP2, COL11A1, BCAT1, FAP, ADAMTS2, MMP2, TREM2, MMP11, GGT5, } \\
\text { SYNDIG1, CLEC11A, COMP, SFRP4, AEBP1, COL1A1, PRRX1, PLXDC2, COL1OA1, COL5A1, POSTN, } \\
\text { ADAMTS7, SULF1, ITGA11, COL6A2, ADAM12, ADAMTS12, SPOCK1, MMP14, MRAS, KIF26B, COL6A3, } \\
\text { COL1A2, FNDC1, CTHRC1, CERCAM, RAB31, COL3A1, ANTXR1, HOPX, OLR1, SPHK1, GAS1, BGN, NTM, } \\
\text { THBS2, PPAPDC1A, COL5A2, C1QTNF5, ZNF469, MFRP, SCARF2 }\end{array}$ & $\begin{array}{l}\text { Connective Tissue Disorders, Organismal Injury and } \\
\text { Abnormalities, Cellular Assembly and Organization }\end{array}$ \\
\hline hsa-miR-6722-5p & RASL12, MAD2L1BP, B3GNT1, SLC25A20 & \\
\hline
\end{tabular}




\begin{abstract}
hsa-miR-934 HECW1, MRC2, SLCIIA1, TIMP2, VCAN, TNC, COL11A1, BCAT1, LZTS1, TRO, MOVIOL1, GLI2, NOTCH3, NUAK1, FAP, EPYC, NOX4, ADAMTS2, MMP2, ZFHX4, MMP11, GGT5, TIMP3, NKAIN4, ISM1, SYNDIG1, MXRA5, WISP1, SYDE1, MEIS3, COMP, WNT2, PCOLCE, SERPINEI, SFRP4, AEBPI, DNM1, COL1A1, COL12AI, MDFI, SPARC, PDGFRB, COL7A1, PRRX1, ST6GALNAC5, MFAP2, RGS4, SPP1, LTBP2, PLXDC2, INHBA, TWIST1, PLAU, COL10A1, GLIS2, A4GALT, KRTI7, ISLR, PXDN, COL5AI, PODNL1, POSTN, LOXL2, ADAMTS7, ANGPTL2, SULF1, ITGAII, BRDT, EMILIN1, ADAMTS14, LUM, HAPLN3, MFGE8, KIFC3, CDH11, RGS16, CTSK, SELENBP1, COL8A1, PDGFC, ADAM12, SERPING1, ODZ4, ADAMTS12, KCNE4, SH2D6, SPOCK1, GUCYIA2, THY1, DKK2, MMP14, DGKI, SPON2, PLXDC1, PDPN, OLFML2B, KIF26B, COL6A3, GPX8, BMPER, COL1A2, FNDC1, CTHRC1, KIAA1462, HTRA1, GPR176, FBN1, GREM1, CERCAM, IGF2, NXN, RAB31, COL3A1, COL22A1, ANTXR1, CDH2, HTRA3, PRKCDBP, HOPX, COL24A1, COL8A2, LRRC15, OLR1, AGR3, PHLDA3, CD248, SPHK1, SOX11, AC100788.1, SSC5D, C3orf80, GAS1, F2R, BGN, NTM, CDR1, THBS2, ZDHHC11, CHSY3, FCGR3A, PPAPDC1A, COL5A2, COL15A1, ATP10A, ODZ3, FAM19A5, PLXNA4, C10orf55, CIQTNF5, ZNF469, MFRP, UGT1A5, SCARF2

hsa-miR-99b-5p CFH, TFPI, PLXNDI, HSPB6, THSD7A, COPZ2, GAS7, MRC2, DCN, IGF1, WWTRI, PHLDBI, SNAI2, GPR124, SAMD4A, NRXN3, FHL1, NLRP2, EHD2, HSF2, NRIH3, VIM, TIMP2, FLT4, VCAN, FAM65A, GPRI24, SAMD4A, NRXN3, FHL1, NLRP2, EHD2, HSF2, NRIH3, VIM, TIMP2, FLT4, VCAN, FAM65A,
STAU2, TNC, EPHA3, PREX2, LMO3, PER3, ELN, CHRDL2, ATP2B4, GUCYIB3, CA11, CDON, CALCRL, MYLK, EMLI, TRAM1, NAV3, STONI-GTF2AIL, ABCC9, RORA, TGFBR3, GNB5, PFN2, POLB, ARHGAPIO, CYBRD1, LIMS2, SPEG, EVC, NDE1, MRVII, FERMT2, FRY, GLI2, NOTCH3, SLC24A1, ZNF532, TACR2, FGFRI, FBLN1, TNRC6C, RUNXIT1, FKBP7, TNS1, MOXD1, CHRNA3, PCDHGA2, KAT6A, BCORL1, AKRIB1, EROILB, MMP2, NID2, CERS4, CCDC80, COL9A3, DPYSL2, SORBS1, NRP1, HCN2, PALM, SUSD2, LGALSI, TTC28, SEPT3, TIMP3, SYNGRI, PAPLN, NFATC4, PLTP, MYL9, MYOM1, CHRDL1, SMARCA1, TIMP1, KATNAL1, C13orf33, ZNF423, CRISPLD2, ZNF174, CORO2B, RASL12, IGDCC4, CTSH, BRF2, PGCP, TRPS1, NUMBL, CLIP3, PTPRS, CARD8, CADM4, CAV2, CAV1, C7orf58, PCOLCE, RARRES2, GLI3, AEBP1, DNM1, MPDZ, ACTA2, MAP3K8, CCL2, CNTNAP1, COL1A1, HDAC5, PMP22, RAB34, MAPK10, TBCID9, TBC1D19, KLHL5, FOLR1, ARHGEF17, LEPREL2, TSPAN11, ACSS3, TENC1, GLI1, MGP, STX2, GPR133, HCFC2, COL12A1, DSE, QKI, PTK7, LAMA4, C7, SPARC, HANDI, ARSB, THBS4, NR3C1, DPYSL3, PDGFRB, CPEB4, DBNI, GNAI2, GNB4, ZBTB47, SLC4A3, ADAM23, KCNIP3, ACVRI, PDEIA, CLIP4, LOXL3, EFEMP1, GLS, IGFBP5, ILIRI, ATF2, WLS, RGS2, OLFML3, NID1, AKT3, ST6GALNAC5, LPHN2, MFAP2, NRP2, CTGF, PKD2, CNRIP1, NKX2-3, PPPIR3C, PYROXD2, MYCT1, PLXDC2, ENOX1, GLT8D2, SCPEP1, TSHZ3, KCNJ8, PSPC1, PDZRN3, CPXM2, LDB3, CALD1, BICC1, EGR2, RASSF8, NECAB1, ITIH5, OBSL1, SLC12A4, PTGIS, TRERF1, RUNX2, C3, C20orf46, GLIS2, AHDC1, RHOJ, ARMCX1, SYNGR3, HIP1, SGCE, FLNC, SNRPN, CGNL1, ISLR, PALLD, CDKNIC, CNN1, KIF1A, PXDN, COL5A1, LAMA5, AKAP12, SYNE1, EDA2R, PDLIM4, GFPT2, AOC3, MAP1B, SERPINF1, DLG4, VPSI3B, MTSSIL, SYTI1, MYH10, DCLK1, POSTN, MYH11, KRBA1, LYVE1, MEIS2, TSPAN2, IL6ST, NAV1, RERG, DZIP1, C5orf13, MDFIC, BAI3, LCA5, ITGA7, NPL, FAM129A, LAMC1, PLXNC1, GPNMB, SCN7A, IL10, CCL21, DNAJB5, SULFI, FXYD6, THBSI STRA6, ZNF280D, ARHGAP29, EMILIN1, DUSP5, CALHM2, ENTPDI, SENP7, CILP, MMRN1, PDE5A, KIAA1644, LUM, WDFY2, FRMD6, SLC38A6, FBLN5, IGF1R, BBS4, MFGE8, TGFB1I1, CDH11, MYOCD, IGFBP4, COL6A1, COL6A2, EMP3, WTIP, HSPG2, CYR61, DPT, RGL1, CTSK, DYRK3, ATP8B2, LYST, WNT9A, ETNK2, RHOB, MEIS1, GPRI7, FAM17IB, PHLDB2, BOC, TRPC1, SLIT2, SCD5, ANK2, SFRP2, OSMR, SSBP2, KCNMB1, PPPIR18, VIP, SDK1, AGBL3, ATP6VIB2, ERLIN2, RGS20, VLDLR, ZEB1, GAS2, SERPING1, ADAM33, TAGLN, JPH2, C10orf68, ARID5B, LATS2, ITPR1, BTBDII, DIP2C, AKAP6, WWC2, LIXIL, KCNE4, HSPB8, SETBP1, SPOCK1, JMY, PLEKHH2, SPARCL1, PELO, LGI2, RBMS1, ASAP1, CACNA2D1, THY1, TBCEL, ABCA6, PGM5, PDLIM3, NCAM2, ADAMTSI, APCDD1, FZD7, GRIP1, KIF5A, C8orf38, RBPMS MMP14, AFAPIL1, TSPAN18, PALM2-AKAP2, MEGF11, MRAS, COLECI2, SLAMF8, CACHD1, CSRP1, CIR, STARD9, SPON2, ZNF208, GPSM1, PLXDC1, ITGA5, AMDHD2, PPAP2B, SDC3, NEXN, VCAM1, DDR2, SNED1, ACTG2, CIQTNF7, COL6A3, C3orf64, FSTL1, LMOD1, IGFBP7, FBLN2, PRICKLE2, CAMK2N2, EMCN, HAND2, GUCY1A3, FAMI98B, CMYA5, COL1A2, DLC1, MED3O, GEM, LETM2, SVEP1, PTCHDI, NLGN4Y, HECTD2, CFL2, ZCCHC24, PHYHIPL, VSTM4, KIAA1462, SALL2, TTC7B, PDZRN4, AMOTL1, NLGN4Y, HECTD2, CFL2, ZCCHC24, PHYHIPL, VSTM4, KIAA1462, SALL2, TTC7B, PDZRN4, AMOTL1,
HTRA1, JAM3, FBNI, LARP6, CLMP, DCHS1, TUB, CDYL2, PRTG, TMX3, MFAP4, AC024270.1, RIMKLB, NGFRAPI, NNMT, C16orf45, RBPMS2, VPS39, GREM1, MAPIA, PBX3, CERCAM, GPRC5B, TBCID2B ZNF180, CORO6, TUBAIA, ZNF528, AXL, PPPIR14A, NXN, IGFBP6, TMEM99, RAB3IL1, LTBP3, PCMTD1, RAB31, SDPR, COL3A1, SEMA4C, LCMT2, CHST14, MN1, ADRB2, SDC2, CLIC4, CCDC8, ZEB2, ANTXRI, LDB2, PCDH7, TPST1, NLGN2, RNF150, DCLK2, DENND5B, LONRF2, COMMD5, SGCD, PYGO1, GRIK1, Clorf190, KCND3, MCC, DSEL, COL24A1, PRNP, LAMB2, CCL11, ID4, NEGR1, ARNT2, SYNPO2, MANEA, EFEMP2, ANUBL1, SLFN11, CYP7B1, NBEA, MRGPRF, BNC2, RHOD, PTPRM, CSPG4, HEG1, CTSF, MSRB3, GLIS1, PODN, FZD4, DES, ARL10, BAIAP2, A2M, ARL4D, TUBB6, TRIL, FZD8, HIC1, PTRF SUMO4, FAM20C, ZNF354C, AC100788.I, DTX3, MAF, CSRNP3, THBD, C17orf57, VWA1, SSC5D, C3orf80, LYNXI, GREM2, CHRM2, TMEM136, ZNF467, MAB2IL2, PLAG1, CHST15, RGMA, SYNM, APIS2, CIS, BGN, MXRA7, NXPH3, CSF1R, IGIP, C21orf90, GJCI, CADM1, SLC8A1, GPC6, TMEM119, CCBE1, FHL3, MEX3B, FBXL7, CBX6, OLFML1, KIRREL, SCFD2, ALDHIA3, PRKD1, PROS1, SLC24A3, FLRT2, AHNAK2, PBX1, ZFP36L1, MORF4L1, RASA3, WWOX, NUDT17, GNG2, PDE2A, ZDHHC17, MITF, DYNC2H1, BCAM, C11orf96, MAGEH1, TRPV2, LDLRAD2, COL14A1, Clorf204, S100A3, ARL4C, PRELP, APOD, FAM180A, C8orf76, FAT4, ZNF565, SRGAP2P2, ZNF34, ZNF781, LAMA2, TCF4, ZNF512B, PDLIM7, FLNA, ANXA6 KANK2, PARVA, ZNF667, SIRPA, ADH4, TPM2, PLN, FAN1, LDB1, PLXNB3, ZNF521, RP3-412A9.11, CESI, GPRASPI, DMD, CIorf95, COL5A2, COL15A1, PCDHGA1, NYNRIN, VGLL3, FAM127C, LCAT, HEXA, SNURF, C6orf174, PLXNA4, NPTXR, ARHGAP23, PCDHGC3, AOPI, PCDHGC5, ARHGEF25, TMEFF1, PLEKHO2, AKAP2, PCDHGC4, STON1, SCARF2, ECSCR, C9orf30-TMEFF1, PCDHGA12, RP11-122A3.2, PCDHGB6, PCDHGA5, PCDHGA7, PCDHGA6, PCDHGA8, PCDHGA11, PCDHGB2, PCDHGB4, PCDHGB7, PCDHGB1 PCDHGA3, RP11-728F11.6, RP3-403A15.5, AC005013.1
\end{abstract}

Developmental Disorder, Hereditary Disorder, Immunological Disease; Cellular Development, Connective Tissue Development and Function, Skeletal and Muscular System Development and Function; Cardiovascular System Development and Function, Organismal Development, Tissue Development; Connective Tissue Disorders, Organismal Injury and Abnormalities, Developmental Disorder; Embryonic Development, Organ Development, Organ Morphology; Cellular Assembly and Organization, Cellular Function and Maintenance, Cancer; Cell-To-Cell Signaling and Interaction, Cellular Assembly and Organization, Cellular Function and Maintenance; Developmental Disorder, Organismal Injury and Abnormalities, Embryonic Development; Cardiovascular System Development and Function, Organ Morphology, Skeletal and Muscular System Development and Function; Organismal Development, Cancer Organismal Injury and Abnormalities; Cancer, Organismal Injury and Abnormalities, Reproductive System Disease; Cellular Development, Cellular Growth and Proliferation, Digestive System Development and Function; Cellular Development, Connective Tissue Development and Function, Embryonic Developve Tis Hevenic Abnormalities, Embryonic Development

study given the smaller sample size of rectal cancer cases and the low frequency of exposure associated with the infrequently expressed miRNAs.

Given their infrequent expression, many of the miRNAs evaluated in our study have no previously recorded association with CRC stage or survival. However, our findings suggest that when highly expressed in a tumor, some infrequently expressed miRNAs have a potentially meaningful association with CRC progression and prognosis as indicated by disease stage and survival.
Interestingly, our analysis of the mRNA expression showed that most associated genes were up-regulated with higher levels of miRNA expression in tumors than normal mucosa (Table 5). This may be counterintuitive, as miRNAs are traditionally thought to post-transcriptionally down-regulate gene expression. In addition to their role in feedback loops, many transcription factors and miRNAs are known to function in feed-forward loops (FFL) in which a given transcription factor (TF) simultaneously up-regulates or down-regulates both the miRNA and the 
mRNA that it targets [3]. This type of FFL is known as an 'incoherent' FFL, as the effect that the miRNA has on the target gene is opposite that of the TF on the target gene. As such, the miRNA and mRNA expression may appear directly associated, because the increase in transcription of the target gene by the TF can outstrip the repression of the mRNA by the miRNA. Posttranscriptional up-regulation is observed in certain cell types, such as developing germ cells, and with certain transcription factors [13]. One such FFL described in $\mathrm{T}$ cell acute lymphoblastic leukemia (T-ALL), involves the NFKB signaling pathway. In this FFL, NFאB activates both $C Y L D$, a tumor suppressor that inhibits the nuclear translocation of NFKB, and miR-19 which suppresses $C Y L D$. If NFkB upregulates miR-19, relative to $C Y L D$, this pathway ultimately allows for the sustained activation of NFKB in T-ALL through the miR19 mediated inhibition of $C Y L D$ [14]. The NFKB complex was one of our top networks associated with genes associated with these miRNAs.

We found that some miRNAs are associated with both stage and survival. Previously, high levels of miR-31 expression were shown to have an association with larger tumor size, poor differentiation, and advanced disease stage [15]. Moreover, high levels of miR-31 expression were associated with poorer survival [15]. This is consistent with our findings that high levels of miR-31-5p expression is associated with both CRC advanced AJCC stage and a greater likelihood of dying of CRC. Runt related transcription factor 2 (RUNX2) was associated with up-regulation by miR-99b-5p in our data. RUNX2 has been associated with Dukes staging, including liver and lymph node metastasis [16]. Additionally, we previously showed that genetic variation in $R U N X 2$, a member of the TGF-b pathway, had prognostic implications [17].

To help interpret the functionality of the infrequently associated miRNAs, we linked them to gene expression data in our colorectal samples. Evaluation of genes that were either up- or down-regulated when these infrequently expressed miRNAs were expressed at higher levels, helped to identify disease functions and networks through which these miRNAs could be operating either directly or indirectly. Further examination of the top networks provides support for the functions of these miRNAs in cancer progression and prognosis. For instance, the main gene in Network 1 translates to Histone 3 (H3), a protein that comprises chromatin nucleosomes. Epigenetic methylation of various amino acids on $\mathrm{H} 3$ allows for increased or decreased expression of both oncogenic or tumor suppressing pathways. For example, H3K27-3me, or trimethylation of the 27th lysine residue, is found in aggressive CRC [18]. One mechanism proposed to explain the oncogenic behavior suggests that polycomb-targeted gene family members $(\mathrm{PcG})$ methylate $\mathrm{H} 3$, thereby downregulating tumor suppressor genes, such as Hand1, which

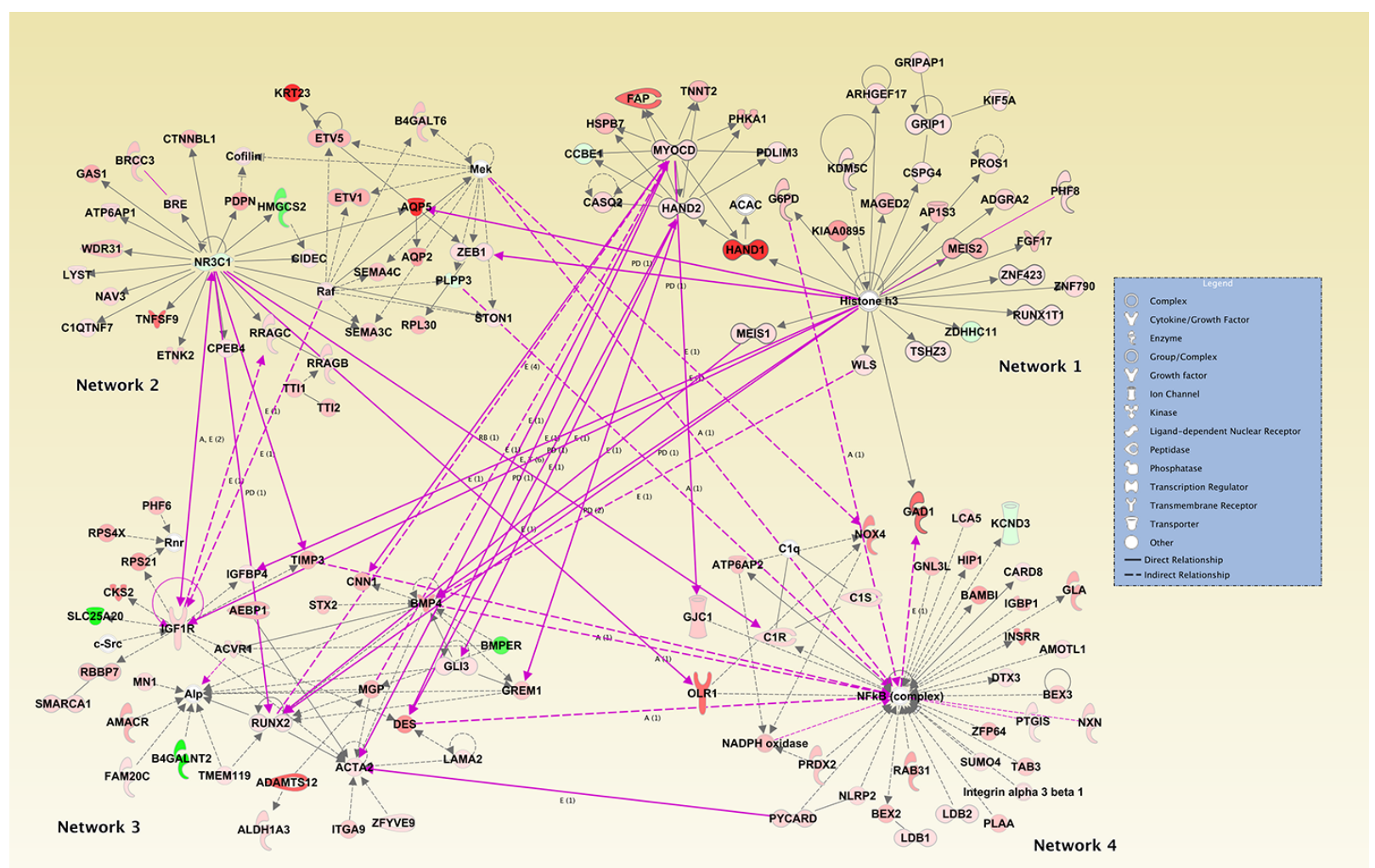

Figure 1: Top four IPA networks associated with genes linked to miRNAs that influence advanced disease stage or survival. 
Table 6: IPA networks derived from genes associated with miRNAs that influenced CRC survival

\begin{tabular}{|c|c|c|c|c|}
\hline Network & Molecules in Network & Score & Focus Molecules & Top Diseases and Functions \\
\hline 1 & $\begin{array}{l}\text { ACAC,ADGRA2,AP1S3,ARHGEF17,CASQ2,CCBE1,CSPG4,FAP,FGF17,G6PD,GAD1,GRIP1,GR } \\
\text { IPAP1,HAND1,HAND2,Histoneh3,HSPB7,KDM5C,KIAA0895,KIF5A,MAGED2,MEIS1,MEIS2,M } \\
\text { YOCD,PDLIM3,PHF8,PHKA1,PROS1,RUNX1T1,TNNT2,TSHZ3,WLS,ZDHHC11,ZNF423,ZNF790 }\end{array}$ & 35 & 33 & $\begin{array}{l}\text { Nervous System Development and Function, } \\
\text { Organ Morphology, Organismal Development }\end{array}$ \\
\hline 2 & $\begin{array}{l}\text { AQP2,AQP5,ATP6AP1,B4GALT6,BRCC3,BRE,C1QTNF7,CIDEC,Cofilin,CPEB4,CTNNBL1,ETNK } \\
\text { 2,ETV1,ETV5,GAS1,HMGCS2,KRT23,LYST,Mek,NAV3,NR3C1,PDPN,PLPP3,Raf,RPL30,RRAGB } \\
\text {,RRAGC,SEMA3C,SEMA4C,STON1,TNFSF9,TTI1,TTI2,WDR31,ZEB1 }\end{array}$ & 33 & 32 & $\begin{array}{l}\text { Lipid Metabolism, Molecular Transport, Small } \\
\text { Molecule Biochemistry }\end{array}$ \\
\hline 3 & $\begin{array}{l}\text { ACTA2,ACVR1,ADAMTS12,AEBP1,ALDH1A3,Alp,AMACR,B4GALNT2,BMP4,BMPER,c-Src,C } \\
\text { KS2,CNN1,DES,FAM20C,GL13,GREM1,IGF1R,IGFBP4,ITGA9,LAMA2,MGP,MN1,PHF6,RBBP7, } \\
\text { Rnr,RPS21,RPS4X,RUNX2,SLC25A20,SMARCA1,STX2,TIMP3,TMEM119,ZFYVE9 }\end{array}$ & 33 & 32 & $\begin{array}{l}\text { Cellular Development, Connective Tissue } \\
\text { Development and Function, Skeletal and } \\
\text { Muscular System Development and Function }\end{array}$ \\
\hline 4 & $\begin{array}{l}\text { AMOTL1,ATP6AP2,BAMBI,BEX2,BEX3,C1q,C1R,C1S,CARD8,DTX3,GJC1,GLA,GNL3L,HIP1,IG } \\
\text { BP1,INSRR,Integrin alpha } 3 \text { beta 1,KCND3,LCA5,LDB1,LDB2,NADPH oxidase, NFkB (complex), } \\
\text { NLRP2,NOX4,NXN,OLR1,PLAA,PRDX2,PTGIS,PYCARD,RAB31,SUMO4,TAB3,ZFP64 }\end{array}$ & 31 & 31 & $\begin{array}{l}\text { Developmental Disorder, Hereditary Disorder, } \\
\text { Immunological Disease }\end{array}$ \\
\hline 5 & $\begin{array}{l}\text { A2M,AKR1B1,amylase,ANPEP,APCDD1,AQP1,ARL4C,CCDC80,CEBPA,CES1,CYP2B6,FHL1,F } \\
\text { ZD7,GG, GJB1,HDL,HSPA4L,HSPH1,KCNMA1,KCNMB1,LCAT,MIOX,MMRN1,Nr1h,OTC,PLX } \\
\text { ND1,PPP1R14A, } \\
\text { PTK7,SERPINF1,SPRR3,THBS1,TRPS1,VGLL3,VLDL-cholesterol,VLDLR }\end{array}$ & 31 & 31 & $\begin{array}{l}\text { Cardiovascular System Development and } \\
\text { Function, Organismal Development, Visual } \\
\text { System Development and Function }\end{array}$ \\
\hline 6 & $\begin{array}{l}\text { ABCB6,ADAM12,AHNAK2,C11orf96,CCL2,CCL11,CDKN1C,CDX2,Ctbp,CYP39A1,DKK2,EVC,F } \\
\text { OXQ1,GLI1,GLI2,GREM2,HIC1,KRT17,LZTS1,MTMR1,NRXN3,PDLIM7,PDZRN3,PI3K (family), } \\
\text { REG4,SERPINE1,SLC11A1,Smad,Smad2/3,SPARCL1,SPP1,SYNE1,TCF4,WWTR1,XIAP }\end{array}$ & 31 & 31 & $\begin{array}{l}\text { Cellular Movement, Cellular Development, } \\
\text { Cellular Growth and Proliferation }\end{array}$ \\
\hline 7 & $\begin{array}{l}\text { ADAM33,ADAMTS2,Alpha catenin,C3orf80,CD99,Cg,COL5A1,COL5A2,COL6A1,COL6A2, } \\
\text { COL6A3,DCHS1,DPT,EPHA3, estrogen receptor,FAM198B,GGCT,HOPX,KCNJ3,LRRN3, LUM, } \\
\text { MEGF10,MMP11,NPL,NRP1,OSMR,PCDH7,PDE5A,PLAU,RGS2,TENM4,TMEFF1,TNC,TRO,Vegf }\end{array}$ & 31 & 31 & $\begin{array}{l}\text { Connective Tissue Disorders, Organismal Injury } \\
\text { and Abnormalities, Cell Morphology }\end{array}$ \\
\hline 8 & $\begin{array}{l}\text { ADAMTS1,ARHGEF25,CALCRL,COL10A1,CTGF,DGKI,DNM1,EFEMP2,ELN,EMCN,EMILIN1, } \\
\text { ERK1/2,FBLN1,FBLN2,FBLN5,FBN1,FEZF1,GPSM1,GRK3,ITGA7,LTBP3,MFGE8,NEU1,NKX2- } \\
\text { 3,NOP16,NPHP1,PFN2,Rock,SH3GL2,SMAD1/5,Smad1/5/8,Sphk,SULF1,TNS1,VRK2 }\end{array}$ & 29 & 30 & $\begin{array}{l}\text { Tissue Development, Connective Tissue } \\
\text { Disorders, Dermatological Diseases and } \\
\text { Conditions }\end{array}$ \\
\hline 9 & $\begin{array}{l}\text { Actin,ACTL6A,BTBD11,CCNB1,DCTPP1,DLG4,EIF1AX,FHL3,FMR1,GRIK5,HCN2,His } \\
\text { tone h4,HTATSF1,KIF1A,LRRC8D,mediator,MORF4L1,MORF4L2,NEGR1,NLGN2,Notch } \\
\text {,PBX3,PCDHGC3,PDHA1,POLB,RNA polymerase II,SLC25A5,SMYD5,SNAI2,SRFBP1, } \\
\text { SUCLA2,TRPV6,TUBB6,TXLNG, VPS39 }\end{array}$ & 29 & 30 & $\begin{array}{l}\text { Cell-To-Cell Signaling and Interaction, Cellular } \\
\text { Assembly and Organization, Cellular Function } \\
\text { and Maintenance }\end{array}$ \\
\hline 10 & $\begin{array}{l}\text { Akt,atypical protein kinase C,CAV2,CHRNA3,COL7A1,CSE1L, CTTNBP2,CYR61, DCBLD2, } \\
\text { DCLK1, ECSCR, FZD8,GEM,IGFBP5,MID1,Muscarinic cholinergic receptor, PDCD10, PHLDB1, } \\
\text { Ppp2c, PREX2,PRKCDBP,PTRF,RAB27B,SCD5,SDC2,SDPR,STRIP2,SYNM,TEAD,TFAP2A,UX } \\
\text { S1,WDFY2,WIF1,Wnt,ZEB2 }\end{array}$ & 27 & 29 & $\begin{array}{l}\text { Cellular Assembly and Organization, Cellular } \\
\text { Function and Maintenance, Cell Morphology }\end{array}$ \\
\hline 11 & $\begin{array}{l}\text { ATRX,CDH2,CLEC11A,Collagen type I,Collagen type IV,EMP3,FLNC,FLT4,Jnk,KALRN, KIF4A, } \\
\text { Ku,LIG4,MAP1LC3A,MRC2,MYBL1,NCAPG2,OSBP2,PARP,PIWIL1,PMP22,POSTN,PP1 protein } \\
\text { complex group,PROCR,PTPRM,RLIM,SENP7,SGCD,SGCE,SMC2,SORBS1,SUV39H1,THY1,VI } \\
\text { M,WRN }\end{array}$ & 27 & 29 & $\begin{array}{l}\text { Cell Morphology, Cell Cycle, Cellular Assembly } \\
\text { and Organization }\end{array}$ \\
\hline 12 & $\begin{array}{l}\text { ACTG2,ADAMTS7,ASAP1,CA11,CBX6,CDK16,COMP,CRISPLD2,DYRK3,FNDC1,FSH,GPC6 } \\
\text {,GPRC5B,HDAC5,hemoglobin,HOXC6,Lh,MFAP2,MYRF,N-cor,OLFML2B,PGR,PRKX,PRRX1, } \\
\text { RGL1,RHOB,SERPING1,SRC (family),STAT5a/b,STRA6,STS,TBL1X,TRERF1,VCAM1,VCAN }\end{array}$ & 27 & 29 & $\begin{array}{l}\text { Cardiovascular Disease, Connective Tissue } \\
\text { Development and Function, Tissue Development }\end{array}$ \\
\hline 13 & $\begin{array}{l}\text { ADCY,ADRB2,AMOT,ANTXR1,ANTXR2,ATP2B4,Beta Arrestin,Calcineurin A,Calcineurin pro } \\
\text { tein(s),CCL21,CDH11,CHRM2,COL11A1,DAPK1,DDR2,EFNB1,EPHB6,ERK,GLRX3,HYAL2 } \\
\text {,JPH2,Mlc,Mmp,MYLK,NFATC4,NMUR2,NONO,NRP2,PDE10A,PDE2A,PLTP,PRDM8,RGS4- } \\
\text { „SLC8A1,WWOX }\end{array}$ & 25 & 28 & $\begin{array}{l}\text { Cancer, Organismal Injury and Abnormalities, } \\
\text { Cardiovascular System Development and } \\
\text { Function }\end{array}$ \\
\hline 14 & $\begin{array}{l}\text { AChR,AIFM1,ASCL2,Calmodulin,CAMK2N2,CaMKII,CASK,COMT,CORO6,cytochrome-c oxid } \\
\text { ase,DTNB,EPHA4,GLRX2,GPC4,HOXA13,IGF1,ITGA11,KANK2,LARP6,MED14,Mitochondrial } \\
\text { complex 1,NCAM2,NECAB1,NUAK1,PDGF-AA,PTPRS,RIMBP2,Sapk, SDC3,SNTA1,SOX8,TAF1, } \\
\text { TFF1,TFPI, TSPAN2 }\end{array}$ & 25 & 28 & $\begin{array}{l}\text { Amino Acid Metabolism, Post-Translational } \\
\text { Modification, Small Molecule Biochemistry }\end{array}$ \\
\hline 15 & $\begin{array}{l}\text { AKT3,Alpha Actinin,Alpha tubulin,ANLN,APOD,BAIAP2, BARX2,BCAT1,C1GALT1C1, } \\
\text { CETN2,Ck2,DPYSL2,DYNC2H1, DZIP1,ENTPD1,F5, FERMT2,Fibrin,FLNA,FOXC1,GP6,GPIB } \\
\text { IIIA, GRIN2B,GUCY1A2,GUCY1A3,GUCY1B3,IFT52,IFT88,MAP1A,MAP1B,PKD2,Rap1,SSX2 } \\
\text { IP,TCR,VAV3 }\end{array}$ & 25 & 28 & $\begin{array}{l}\text { Cell Morphology, Cellular Assembly and } \\
\text { Organization, Cellular Function and Maintenance }\end{array}$ \\
\hline 16 & $\begin{array}{l}\text { AP1S2,ATP6V1B2,CADM1,CERS4,CFL2,CLCA1,CLIP4,CSRNP3,CSTF1,CYP4F8,DMD,FLRT2,F } \\
\text { OLR1,GBA,GRHL1,HSD17B10,MAP3K9,MPP6,NDE1,NNMT,NSDHL,PIP5K1B,PLK2,PPP1R3C, } \\
\text { RGMA,RORA,S100A3,SELENBP1,SLC13A2,SLC39A5,SLC9A7,Sos,ST3GAL4,ST3GAL6,TTC7B }\end{array}$ & 25 & 28 & $\begin{array}{l}\text { Developmental Disorder, Hereditary Disorder, } \\
\text { Neurological Disease }\end{array}$ \\
\hline 17 & $\begin{array}{l}\text { 14-3-3,ANXA6,ARAF,CDON,Collagen type III,CSF1R,CYP4F3,DRD5,EMD,GLIS2,GPNMB, HD } \\
\text { AC6,Hsp27,HSPB6,IGFBP6,LOXL2,LTBP2,MYH10,MYH11,NELFCD,NOTCH3,P38 MAPK,PBX } \\
\text { 1,Pld,QKI,RANBP1,SAMD4A,Sfk,SIRPA,SMTN,STXBP1,TGFB1I1,TMSB4,tubulin (family),VRK1 }\end{array}$ & 24 & 27 & $\begin{array}{l}\text { Cancer, Organismal Injury and Abnormalities, } \\
\text { Reproductive System Disease }\end{array}$ \\
\hline
\end{tabular}

is another member of Network 1. Demethylation of H3K4 dimethylation has been associated with CRC survival [19]. Additionally, miRNAs have been found to down-regulate PcG family members in a tumor suppressor fashion, significantly decreasing cell viability and migration of CRC cells [20]. Our study shows three miRNAs, miR143-5p, miR-145-3p, miR-99b-5p, that are associated with Hand 1 gene expression and are found to increase the likelihood of dying if highly expressed in CRC. Future studies may implicate the aforementioned miRNAs in the $\mathrm{H} 3$ trimethylation pathway for CRC survival and prognosis.
A central molecule in Network 2 is NR3Cl, a glucocorticoid receptor that induces apoptotic cell death by suppressing expression of anti-apoptotic proteins, such as $B C L 2$ and $M C L 1$, as well as up-regulating expression of pro-apoptotic proteins like BCL2-like apoptosis initiator 11 [21]. Activation of the glucocorticoid receptor has been demonstrated to inhibit invasion and cell migration through disruption of the epithelial-to-mesenchymal transition in hypoxic environments in colon cancer lines [22]. Furthermore, NR3C1 has been proposed as a marker to differentiate between CIMP-high and CIMP- 
low tumors [23]; CIMP status is of potential prognostic significance [24-26]. Our study shows that miR-99b-3p, whose high expression levels increases the likelihood of death in CRC, is associated with the down-regulation of NR3C1. This down-regulation corresponds to previous literature that shows that $\mathrm{NR} 3 \mathrm{C} 1$ can be transcriptionally inactivated in colorectal tumorigenesis [27]. Our findings suggest that the negative survival profile associated with the dysregulation of miR-99b-3p could be in part due to the inhibition of NR3C1.

$I G F 1 R$ is the central molecule in our third IPA pathway. IGF1R is a tyrosine kinase receptor for insulin like growth factors (IGF) 1 and 2, that is commonly overexpressed in CRC [28]. IGF1R is known to induce HIF-1a and VEGF, thus playing an important role in angiogenesis, and has been associated with CRC metastasis [29]. Moreover, crosstalk between the IGF1R and EGFR pathways has been shown to contribute to acquired resistance to EGFR inhibitors and other tyrosine kinase inhibitors [29-31]. IGF1R therefore has prognostic and survival implications for those with CRC and other IGF1R positive tumors. We found that miR-99b-5p upregulates IGF1R and is associated with CRC survival. Thus, our findings are consistent with earlier reports and suggest that IGF1R's association with CRC survival, may, in part, be mediated by infrequently expressed miRNAs.

This study has several strengths and limitations. One of the major strengths is our ability to examine infrequently expressed miRNAs because of our large sample size. Although the sample size is one of the largest available to date, evaluation of these infrequently expressed miRNAs has an impact on the study power. We have used a $Q$-value threshold of 0.15 , however given the number of associations it is likely that over $85 \%$ of them are true findings. We have been able to obtain more information on functionality of these miRNAs by comparing them to our colorectal RNA-Seq data to identify genes whose expression may be associated with these miRNAs. This has enabled us to crudely evaluate molecular pathways through which these miRNAs may be involved and to begin to understand how these miRNAs function. We encourage others to evaluate these miRNAs in both a clinical and laboratory setting to verify their association with survival as well as to gain additional insight into their functionality. Given the dates of diagnosis for these cases, it is possible that new treatment modalities may modify the results we present.

\section{MATERIALS AND METHODS}

\section{Study participants}

Study participants were recruited as part of two population-based case-control studies that included all incident colon and rectal cancer between 30 to 79 years of age who resided in Utah or were of the Kaiser Permanente
Medical Care Program (KPMCP) in Northern California. Participants were non-Hispanic white, Hispanic, or black for the colon cancer study and also included participants of Asian race for the rectal portion of the study [32, 33] Case diagnosis was verified by tumor registry data as a first primary adenocarcinoma of the colon and were diagnosed between October 1991 and September 1994 and for rectal were diagnosed between May 1997 and May 2001. Detailed study methods have been described [9]. The Institutional Review Boards at the University of Utah and at KPMCP approved the study.

\section{RNA processing}

Formalin-fixed paraffin embedded tissue from the initial biopsy or surgery was used to extract RNA. Carcinoma tissue and adjacent normal mucosa were used to make RNA. Cells were dissected from 1-4 sequential sections on aniline blue stained slides using an H\&E slide for reference. Total RNA was extracted, isolated, and purified using the RecoverAll Total Nucleic Acid isolation kit (Ambion); RNA yields were determined using a NanoDrop spectrophotometer.

\section{miRNA}

The Agilent Human miRNA Microarray V19.0 was used. The microarray contains probes for 2006 unique human miRNAs as described previously. Data were required to pass stringent $\mathrm{QC}$ parameters established by Agilent that included tests for excessive background fluorescence, excessive variation among probe sequence replicates on the array, and measures of the total gene signal on the array to assess low signal. If samples failed to meet quality standards for any of these parameters, the sample was re-labeled, hybridized to arrays, and re-scanned. If a sample failed $\mathrm{QC}$ assessment a second time, the sample was deemed to be of poor quality and the sample was excluded from analysis. We excluded 60 carcinoma and 80 normal samples that failed QC [9]. Our previous analysis has shown that the repeatability associated with this microarray was extremely high $(r=0.98), 21$ and that comparison of miRNA expression levels obtained from the Agilent microarray to those obtained from qPCR had an agreement of $100 \%$ in terms of directionality of findings and that the fold change calculated for the miRNA expression difference between carcinoma and normal colonic mucosa was almost identical [10]. Of the 2006 unique human miRNAs assessed on the Agilent microarray, 1247 were expressed in colon carcinoma tissue, 1234 in normal colon mucosa, and 1147 miRNAs were expressed in both colon carcinoma and normal mucosa; 1124 miRNAS were expressed in rectal carcinoma, 1136 miRNAs were expressed in rectal normal mucosa and 1068 miRNAs were expressed in both rectal carcinoma and normal 
mucosa. Of the 1893 carcinoma/normal pairs available for analysis 31 were excluded from survival analysis because of lacking survival information.

To normalize differences in miRNA expression that could be attributed to the array, amount of RNA, location on array, or factors that could erroneously influence miRNA expression levels, total gene signal was normalized by multiplying each sample by a scaling factor which was the median of the 75th percentiles of all the samples divided by the individual 75 th percentile of each sample [34].

\section{mRNA: RNA-Seq sequencing library preparation and data processing}

Total RNA from 245 colorectal carcinoma and normal mucosa pairs was chosen for sequencing; 217 pairs passed quality control and are used in these analyses to identify mRNAs associated with infrequently expressed miRNAs. These samples were taken from the study subjects used for miRNA analysis and were extracted, isolated and purified as previously described [35]. RNA library construction was done with the Illumina TruSeq Stranded Total RNA Sample Preparation Kit with Ribo-Zero. The samples were then fragmented and primed for cDNA synthesis, adapters were then ligated onto the cDNA, and the resulting samples were then amplified using PCR; the amplified library was then purified using Agencount AMPure XP beads. A more detailed description of the methods can be found in our previous work [36]. Illumina TruSeq v3 single read flow cell and a 50 cycle single-read sequence run was performed on an Illumina HiSeq instrument. Reads were aligned to a sequence database containing the human genome (build GRCh37/hg19, February 2009 from genome.ucsc.edu) and alignment was performed using novoalign v2.08.01. Counts were calculated for each exon and UTR of the genes using a list of gene coordinates obtained from http://genome.ucsc.edu. Total gene counts were determined. We dropped genes that were not expressed in our RNA-Seq data or for which the expression was missing for the majority of samples [36].

\section{Survival and stage information}

Cancer survival information was obtained from local cancer registries in Utah and California, both of which are SEER (Surveillance, Epidemiology, and End Results) cancer registries. Information on date of diagnosis and date of last contact or death were obtained in order to calculate survival months. Cause of death along with AJCC staging, based on SEER summary stage data in conjunction with pathology reports, were obtain obtained.

\section{Statistical methods}

The study focuses on infrequently expressed miRNAs which we define as being expressed in less than $50 \%$ of the study population for either normal mucosa or tumor. To be included in the analysis, miRNAs had to have a mean level of expression of 1.0 Agilent Relative Florescent Unit (ARFU) in carcinoma or normal mucosa (to avoid low levels of background noise) and be expressed in at least 30 individuals in order to be able to evaluate survival. For reference, miRNAs expressed in over $50 \%$ of the population have a median level of expression of 30.32 ARFUs with a range of 0.18 to over 47,000 ARFU. A total of 304 miRNAs were analyzed that fit these criteria (Supplementary Table 1 provides detailed information on all of the infrequently expressed miRNAs in either colon or rectal tissue). We used paired carcinoma and normal mucosa miRNA expression, evaluating differential expression between the two tissue types to control for differences in expression by tumor site and other potential confounding factors. Each infrequently expressed miRNA could be considered expressed or not in each of tumor and normal, resulting in three primary dysregulation groups based on the tumor-normal expression differences: up-regulated (expressed more in tumor than in normal), down-regulated (expressed more in normal than in tumor), and referent (neither up- nor down-regulated beyond the 25 th or 75 th percentile). Rather than forcing the same number of subjects to fall into these three groups for all infrequently expressed miRNAs, cutpoints were selected based on the upper $25 \%$ and lower $25 \%$ of the tumornormal differences for all infrequently expressed miRNAs. The resulting three-level dysregulation group factor (up, down, or referent) was used as a predictor in a per-miRNA Cox proportional hazard ratio (HR) model also adjusting for age, study center, AJCC Stage, and sex when evaluating survival. Further adjustment for MSI tumor status had minimal effect on survival associations, with hazard ratios changing less than 0.01 and $p$ values being basically unaltered. Analyses were run separately for cases diagnosed with all CRC, colon cancer specifically, and rectal cancer specifically; additional adjustment for tumor site for overall CRC analysis did not appreciatively alter the results. We analyzed differences in infrequent miRNA expression between those diagnosed at AJCC stages 1 and 2 compared to those diagnosed at more advanced stages (AJCC stages 3 and 4) utilizing a logistic regression analysis adjusting for age, study center, and sex. Disease stages were combined to increase power and results from stage 1 and 2 were not significantly different. Adjustment for multiple comparisons was done using the positive false discovery rate $Q$ value [37]; given the infrequent expression of these miRNAs, we report any associations for which the $Q$ value was less than or equal to 0.15 ; a $Q$ value of 0.15 would imply that up to $15 \%$ of findings could be expected to be false. We believe that these miRNAs could merit further study. 
We evaluated those miRNA with a HR dysregulation group $Q$ value of $\leq 0.15$ (24 miRNAs) with RNA-Seq data to determine genes whose mRNA expression levels were associated with these less frequently expressed miRNAs. To determine statistical significance, we ran a Fisher-Pitman Monte Carlo test with 10,000 permutations comparing mean levels of tumor-normal gene expression differences across miRNA dysregulation groups ( $<75$ th\%tile vs $>75$ th\%tile) in R using the 'coin' package [38] and applied a false discovery rate (FDR) of $<0.05$ to adjust for multiple comparisons. The RPKM (Reads per Kilobase per Million) mRNA expression level data were used in these analyses. Ingenuity Pathway Analysis ${ }^{\circledR}$ (IPA) was used to determine specific pathways that could be involved with genes regulated by these miRNAs with a highly stringent score criterions of 20 required; the score is calculated as $-\log 10 \mathrm{P}$, where $\mathrm{P}$ is generated by a Fisher's exact test $[39,40]$. Studies have found scores $>3$ to be significant, with a score of 3 indicating a $1 / 1000$ chance that the focus genes are in a network due to random chance [41-43]. Other studies have opted to utilize more stringent criteria and higher scores to ensure that their discovered networks are highly significant $[40,44]$; we utilized highly stringent criteria, only including networks with scores over 20.

\section{CONCLUSIONS}

Our large study of population-based cases of CRC enables us to evaluate associations between infrequently expressed miRNAs and stage and survival. Our data suggest that miRNAs that are infrequently expressed in the population, when expressed at high levels in CRC tumors may have an impact on metastatic potential, as indicated by disease stage as well as prognosis, as indicated by survival. Since these infrequently miRNAs are generally upregulated, higher levels of their expression in tumors could be a good indicator of prognosis. Confirmation of these findings could lead to a set of miRNAs for evaluation in tumors to help guide treatment given their impact on metastatic potential. Our data also suggest that extremely low levels of miRNA expression may have little biological significance and contribute to background noise. These infrequently expressed miRNAs when expressed at higher levels appear to be associated with expression of genes that have been linked to CRC progression and prognosis.

\section{Abbreviations}

AJCC: American Joint Committee on Cancer; ARFU: Agilent Relative Florescent Units; CRC: Colorectal Cancer; CI: Confidence Interval; FFL: Feed Forward Loop; HR: Hazard Ratio; IPA: Ingenuity Pathway Analysis; KPMCP: Kaiser Permanente Medical Care Program; miRNA: microRNA; RPKM: Reads Per Kilobase per Million; RUNX2: Runt related factor 2;
SEER: Surveillance Epidemiology and End Results; QC: Quality Control.

\section{Author contributions}

MS obtained funding, planned study, oversaw study data collection and analysis, and wrote the manuscript. AP and FL helped interpret findings and helped write the manuscript. JS provided input into the statistical analysis

LM conducted bioinformatics analysis and helped write manuscript. WS reviewed slides. RW oversaw laboratory analysis. JH conducted statistical analysis and managed data. All authors provided input into data interpretation and approved final manuscript.

\section{ACKNOWLEDGMENTS}

The contents of this manuscript are solely the responsibility of the authors and do not necessarily represent the official view of the National Cancer Institute. We acknowledge Sandra Edwards for data oversight and study management, and Michael Hoffman and Erica Wolff for miRNA analysis. We acknowledge Dr. Bette Caan and the staff at the Kaiser Permanente Medical Research Program for sample and data collection.

\section{CONFLICTS OF INTEREST}

None of the authors have financial and non-financial competing interests to report.

\section{FUNDING}

This study was supported by NCI grants CA163683 and CA48998.

\section{REFERENCES}

1. Ambros V. The functions of animal microRNAs. Nature. 2004; 431:350-355.

2. Murray BS, Choe SE, Woods M, Ryan TE, Liu W. An in silico analysis of microRNAs: mining the miRNAome. Mol Biosyst. 2010; 6:1853-1862.

3. Arora S, Rana R, Chhabra A, Jaiswal A, Rani V. miRNAtranscription factor interactions: a combinatorial regulation of gene expression. Mol Genet Genomics. 2013; 288:77-87.

4. Gartel AL, Kandel ES. miRNAs: Little known mediators of oncogenesis. Semin Cancer Biol. 2008; 18:103-110.

5. Nam S, Li M, Choi K, Balch C, Kim S, Nephew KP. MicroRNA and mRNA integrated analysis (MMIA): a web tool for examining biological functions of microRNA expression. Nucleic Acids Res. 2009; 37:W356-362.

6. Drusco A, Nuovo GJ, Zanesi N, Di Leva G, Pichiorri F, Volinia S, Fernandex A, Antenucci A, Costinean S, Bottoni A, Rosito IA, Liu CG, Burch A, et al. MicroRNA 
profiles discriminate among colon cancer metastasis. PLoS One. 2014; 9:e96670.

7. Bandres E, Cubedo E, Agirre X, Malumbres R, Zarate R, Ramirez N, Abajo A, Navarro A, Moreno I, Monzo M, Garcia-Foncillas J. Identification by Real-time PCR of 13 mature microRNAs differentially expressed in colorectal cancer and non-tumoral tissues. Mol Cancer. 2006; 5:29.

8. Xi Y, Formentini A, Chien M, Weir DB, Russo JJ, Ju J, Kornmann M, Ju J. Prognostic Values of microRNAs in Colorectal Cancer. Biomark Insights. 2006; 2:113-121.

9. Slattery ML, Herrick JS, Pellatt DF, Stevens JR, Mullany LE, Wolff E, Hoffman MD, Samowitz WS, Wolff RK. MicroRNA profiles in colorectal carcinomas, adenomas and normal colonic mucosa: variations in miRNA expression and disease progression. Carcinogenesis. 2016; 37:245-261.

10. Pellatt DF, Stevens JR, Wolff RK, Mullany LE, Herrick JS, Samowitz W, Slattery ML. Expression Profiles of miRNA Subsets Distinguish Human Colorectal Carcinoma and Normal Colonic Mucosa. Clin Transl Gastroenterol. 2016; 7:e152.

11. Slattery ML, Herrick JS, Pellatt DF, Mullany LE, Stevens JR, Wolff E, Hoffman MD, Wolff RK, Samowitz W. Sitespecific associations between miRNA expression and survival in colorectal cancer cases. Oncotarget. 2016; 7:60193-60205. https://doi.org/10.18632/oncotarget.11173.

12. Slattery ML, Lee FY, Pellatt AJ, Mullany LE, Stevens JR, Samowitz WS, Wolff RK, Herrick JS. Infrequently expressed miRNAs in colorectal cancer tissue and tumor molecular phenotype. Mod Pathol. 2017; 30:1152-1169.

13. Vasudevan S. Posttranscriptional upregulation by microRNAs. Wiley Interdiscip Rev RNA. 2012; 3:311-330.

14. Zhang HM, Kuang S, Xiong X, Gao T, Liu C, Guo AY. Transcription factor and microRNA co-regulatory loops: important regulatory motifs in biological processes and diseases. Brief Bioinform. 2015; 16:45-58.

15. Nosho K, Igarashi H, Nojima M, Ito M, Maruyama R, Yoshii S, Naito T, Sukawa Y, Mikami M, Sumioka W, Yamamoto E, Kurokawa S, Adachi Y, et al. Association of microRNA-31 with BRAF mutation, colorectal cancer survival and serrated pathway. Carcinogenesis. 2014; 35:776-783.

16. Sase T, Suzuki T, Miura K, Shiiba K, Sato I, Nakamura Y, Takagi K, Onodera Y, Miki Y, Watanabe M, Ishida K, Ohnuma S, Sasaki H, et al. Runt-related transcription factor 2 in human colon carcinoma: a potent prognostic factor associated with estrogen receptor. Int J Cancer. 2012; 131:2284-2293.

17. Slattery ML, Lundgreen A, Herrick JS, Wolff RK, Caan BJ. Genetic variation in the transforming growth factor-beta signaling pathway and survival after diagnosis with colon and rectal cancer. Cancer. 2011; 117:4175-4183.

18. Tan J, Yang X, Jiang X, Zhou J, Li Z, Lee PL, Li B, Robson P, Yu Q. Integrative epigenome analysis identifies a Polycomb-targeted differentiation program as a tumor- suppressor event epigenetically inactivated in colorectal cancer. Cell Death Dis. 2014; 5:e1324.

19. Tamagawa H, Oshima T, Shiozawa M, Morinaga S, Nakamura Y, Yoshihara M, Sakuma Y, Kameda Y, Akaike M, Masuda M, Imada T, Miyagi Y. The global histone modification pattern correlates with overall survival in metachronous liver metastasis of colorectal cancer. Oncol Rep. 2012; 27:637-642.

20. Vishnubalaji R, Hamam R, Abdulla MH, Mohammed MA, Kassem M, Al-Obeed O, Aldahmash A, Alajez NM. Genome-wide mRNA and miRNA expression profiling reveal multiple regulatory networks in colorectal cancer. Cell Death Dis. 2015; 6:e1614.

21. Wei TT, Lin YT, Chen WS, Luo P, Lin YC, Shun CT, Lin YH, Chen JB, Chen NW, Fang JM, Wu MS, Yang KC, Chang LC, et al. Dual Targeting of 3-Hydroxy3-methylglutaryl Coenzyme A Reductase and Histone Deacetylase as a Therapy for Colorectal Cancer. E Bio Medicine. 2016; 10:124-136.

22. Kim JH, Hwang YJ, Han SH, Lee YE, Kim S, Kim YJ, Cho JH, Kwon KA, Kim JH, Kim SH. Dexamethasone inhibits hypoxia-induced epithelial-mesenchymal transition in colon cancer. World J Gastroenterol. 2015; 21:9887-9899.

23. Ahlquist T, Lind GE, Costa VL, Meling GI, Vatn M, Hoff GS, Rognum, TO, Skotheim RI, Thiis-Evensen E, Lothe RA. Gene methylation profiles of normal mucosa, and benign and malignant colorectal tumors identify early onset markers. Mol Cancer. 2008; 7:94.

24. Kim CH, Huh JW, Kim HR, Kim YJ. CpG island methylator phenotype is an independent predictor of survival after curative resection for colorectal cancer: A prospective cohort study. J Gastroenterol Hepatol. 2017; 32:1469-1474.

25. Min BH, Bae JM, Lee EJ, Yu HS, Kim YH, Chang DK, Kim HC, Park CK, Lee SH, Kim KM, Kang GH. The $\mathrm{CpG}$ island methylator phenotype may confer a survival benefit in patients with stage II or III colorectal carcinomas receiving fluoropyrimidine-based adjuvant chemotherapy. BMC Cancer. 2011; 11:344.

26. Van Rijnsoever M, Elsaleh H, Joseph D, McCaul K, Iacopetta B. $\mathrm{CpG}$ island methylator phenotype is an independent predictor of survival benefit from 5-fluorouracil in stage III colorectal cancer. Clin Cancer Res. 2003; 9:2898-2903.

27. Lind GE, Kleivi K, Meling GI, Teixeira MR, Thiis-Evensen E, Rognum TO, Lothe RA. ADAMTS1, CRABP1, and NR3C1 identified as epigenetically deregulated genes in colorectal tumorigenesis. Cell Oncol. 2006; 28:259-272.

28. Li B, Ge L, Li M, Wang L, Li Z. miR-448 suppresses proliferation and invasion by regulating IGF1R in colorectal cancer cells. Am J Transl Res. 2016; 8:3013-3022.

29. Shali H, Ahmadi M, Kafil HS, Dorosti A, Yousefi M. IGF1R and c-met as therapeutic targets for colorectal cancer. Biomed Pharmacother. 2016; 82:528-536. 
30. Halasz M, Kholodenko BN, Kolch W, Santra T. Integrating network reconstruction with mechanistic modeling to predict cancer therapies. Sci Signal. 2016; 9:ra114.

31. Seow HF, Yip WK, Fifis T. Advances in targeted and immunobased therapies for colorectal cancer in the genomic era. Onco Targets Ther. 2016; 9:1899-1920.

32. Slattery ML, Potter J, Caan B, Edwards S, Coates A, Ma KN, Berry TD. Energy balance and colon cancer-beyond physical activity. Cancer research. 1997; 57:75-80.

33. Slattery ML, Caan BJ, Benson J, Murtaugh M. Energy balance and rectal cancer: an evaluation of energy intake, energy expenditure, and body mass index. Nutr Cancer. 2003; 46:166-171.

34. Agilent Technologies I. Agilent GeneSpring User Manual, vol. 2015. Aglient Technologies Inc: Santa Clara, CA, 2013.

35. Slattery ML, Herrick JS, Mullany LE, Valeri N, Stevens J, Caan BJ, Samowitz W, Wolff RK. An evaluation and replication of miRNAs with disease stage and colorectal cancer-specific mortality. Int J Cancer. 2015; 137:428-438.

36. Slattery ML, Pellatt DF, Mullany LE, Wolff RK, Herrick JS. Gene expression in colon cancer: A focus on tumor site and molecular phenotype. Genes, chromosomes \& cancer. 2015; 54:527-541.

37. Storey JD. A direct approach to false discovery rates. Journal of the Royal Statistical Society: Series B. 2002; 64S:479-498.

38. Torsten Horthorn KH, van de Wiel MA, Zeileis A. Implementing a Class of Permutation Tests: The Coin Package. Journal of Statistical Software. 2008; 28:1-23.
39. Li Y, Carrillo JA, Ding Y, He Y, Zhao C, Zan L, Song J. Ruminal Transcriptomic Analysis of Grass-Fed and GrainFed Angus Beef Cattle. PLoS One. 2015; 10:e0116437.

40. Jia P, Kao CF, Kuo PH, Zhao Z. A comprehensive network and pathway analysis of candidate genes in major depressive disorder. BMC Syst Biol. 2011; 5:S12.

41. Baranzini SE, Galwey NW, Wang J, Khankhanian P, Lindberg R, Pelletier D, Wu W, Uitdehaag BM, Kappos L; GeneMSA Consortium, Polman CH, Matthews PM, Hauser SL, et al. Pathway and network-based analysis of genomewide association studies in multiple sclerosis. Human molecular genetics. 2009; 18:2078-2090.

42. Yan-Fang T, Dong W, Li P, Wen-Li Z, Jun L, Na W, Jian W, Xing F, Yan-Hong L, Jian N, Jian P. Analyzing the gene expression profile of pediatric acute myeloid leukemia with real-time PCR arrays. Cancer cell international. 2012; 12:40.

43. Naito Y, Kuroda M, Mizushima K, Takagi T, Handa O, Kokura S, Yoshida N, Ichikawa H, Yoshikawa T. Transcriptome Analysis for Cytoprotective Actions of Rebamipide against Indomethacin-Induced Gastric Mucosal Injury in Rats. Journal of clinical biochemistry and nutrition. 2007; 41:202-210.

44. Reyes-Gibby CC, Yuan C, Wang J, Yeung SC, Shete S. Gene network analysis shows immune-signaling and ERK1/2 as novel genetic markers for multiple addiction phenotypes: alcohol, smoking and opioid addiction. BMC systems biology. 2015; 9:25. 